Draft version August 24, 2018

Preprint typeset using $\mathrm{IAT}_{\mathrm{E}} \mathrm{X}$ style emulateapj v. 5/2/11

\title{
THE DEUTERIUM FRACTIONATION TIMESCALE IN DENSE CLOUD CORES: A PARAMETER SPACE EXPLORATION
}

\author{
SHuO KOnG ${ }^{1}$ \\ Dept. of Astronomy, University of Florida, Gainesville, Florida 32611, USA \\ Paola Caselli ${ }^{2,3}$ \\ Max-Planck-Institute for Extraterrestrial Physics (MPE), Giessenbachstr. 1, D-85748 Garching, Germany and \\ School of Physics and Astronomy, University of Leeds, Leeds LS2 9JT, UK \\ Jonathan C. TAN ${ }^{1,4}$ \\ Dept. of Astronomy, University of Florida, Gainesville, Florida 32611, USA and \\ Dept. of Physics, University of Florida, Gainesville, Florida 32611, USA \\ VALENTINE WAKELAM ${ }^{5,6}$ \\ University of Bordeaux, LAB, UMR 5804, 33270, Floirac, France and \\ CNRS, LAB, UMR 5804, 33270, Floirac, France \\ AND \\ Olli SipILÄ ${ }^{2}$ \\ Max-Planck-Institute for Extraterrestrial Physics (MPE), Giessenbachstr. 1, D-85748 Garching, Germany \\ Draft version August 24, 2018
}

\begin{abstract}
The deuterium fraction $\left[\mathrm{N}_{2} \mathrm{D}^{+}\right] /\left[\mathrm{N}_{2} \mathrm{H}^{+}\right]$, may provide information about the ages of dense, cold gas structures, important to compare with dynamical models of cloud core formation and evolution. Here we introduce a complete chemical network with species containing up to three atoms, with the exception of the Oxygen chemistry, where reactions involving $\mathrm{H}_{3} \mathrm{O}^{+}$and its deuterated forms have been added, significantly improving the consistency with comprehensive chemical networks. Deuterium chemistry and spin states of $\mathrm{H}_{2}$ and $\mathrm{H}_{3}^{+}$isotopologues are included in this primarily gas-phase chemical model. We investigate dependence of deuterium chemistry on model parameters: density $\left(n_{\mathrm{H}}\right)$, temperature, cosmic ray ionization rate, and gas-phase depletion factor of heavy elements $\left(f_{\mathrm{D}}\right)$. We also explore the effects of time-dependent freeze-out of gas-phase species and dynamical evolution of density at various rates relative to free-fall collapse. For a broad range of model parameters, the timescales to reach large values of $D_{\text {frac }}^{\mathrm{N}_{2} \mathrm{H}^{+}} \gtrsim 0.1$, observed in some low- and high-mass starless cores, are relatively long compared to the local free-fall timescale. These conclusions are unaffected by introducing time-dependent freeze-out and considering models with evolving density, unless the initial $f_{\mathrm{D}} \gtrsim 10$. For fiducial model parameters, achieving $D_{\text {frac }}^{\mathrm{N}_{2} \mathrm{H}^{+}} \gtrsim 0.1$ requires collapse to be proceeding at rates at least several times slower than that of free-fall collapse, perhaps indicating a dynamically important role for magnetic fields in the support of starless cores and thus the regulation of star formation.
\end{abstract}

Subject headings: Physical data and processes: astrochemistry - stars: formation - ISM: clouds

\section{INTRODUCTION}

Deuterated molecules are useful diagnostic tools to study the cold and dense environments where stars are born. This has been demonstrated in low-mass starforming regions (e.g., Caselli 2002; Bacmann et al. 2003 Crapsi et al. 2005, 2007. Emprechtinger et al. 2009. Friesen et al. 2010), as well as in regions thought to be precursors of massive stars and stellar clusters (e.g., Fontani et al. 2006, 2009, 2011; Pillai et al. 2007, 2012). Deuterated species can be used to infer the elusive electron fraction $x(e)$ (e.g., Guelin et al. 1977; Wootten et

\begin{tabular}{l}
$\mid$ skong@astro.ufl.edu \\
\hline caseili@mpe.mpg.de \\
jt@astro.ufi.edu \\
wakelam@obs.u-bordeauxi.fr \\
olli.sipila@heisinki.n
\end{tabular}

al. 1979, Caselli et al. 1998, Bergin et al. 1999, Dalgarno 2006, although the equations in these papers need to be modified to include the doubly and triply deuterated forms of $\mathrm{H}_{3}^{+}$) and the age of molecular clouds (Pagani et al. 2011, 2013; Brünken et al. 2014). Electron fraction and cloud age are two important parameters to shed light on the dynamical evolution of star-forming regions, as the ambipolar diffusion timescale is directly proportional to $x(e)$ (e.g., Shu et al. 1987) and the age can put stringent constraints on the mechanism(s) regulating cloud core formation (e.g., magnetic fields, turbulence and shocks). However, variations in cosmic-ray (CR) ionization rate, volume density, kinetic temperature, rates of molecular freeze-out onto dust grain surfaces and the ortho-to-para ratio of $\mathrm{H}_{2}$ make attempts to fix these values rather uncertain, especially for regions with poorly known physical 
structure.

Extensive effort have been spent on understanding the chemistry in starless/pre-stellar cores (e.g. Flower et al. 2006 Pagani et al. 2009, hereafter P09; Parise et al. 2011 Aikawa et al. 2012; Sipilä et al. 2013). However, since they focused on specific aspects of modeling, they were limited by either the incompleteness of reactions or the narrow range of physical conditions. In this paper we use a complete reduced network with up-to-date rate coefficients, and explore uniformly the parameter space without any prior bias about the dynamical history, as this depends on poorly known physical quantities such as magnetic fields and turbulence. This parameter space exploration is needed to understand the dependence of the chemical composition (in particular the abundance of deuterated molecules) on basic physical properties and parameters, and to help the interpretation of observational data. It is the first time such an exploration has been done with complete spin-state reactions.

Recently, Pagani et al. (2013) investigated these effects by coupling hydrodynamics with chemistry. They developed an astrochemical model to derive the age of low-mass cores and extensively discussed the role of $\mathrm{O}-$ $\mathrm{H}_{2}$. However, they used a relatively limited set of reactions: their chemical network was first based on that of Lesaffre et al. (2005) with 120 reactions and 35 species; then later improved by P09 to include about 400 reactions. The P09 network ignores reactions with rate coefficients below $10^{-15} \mathrm{~cm}^{-3} \mathrm{~s}^{-1}$, thus no radiative association reactions, important for carbon chemistry, are included. Furthermore, their model does not fully track the $\mathrm{N}$ chemistry ( $\mathrm{N}_{2}$ abundance is a parameter), so they are not able to predict absolute abundances of $\mathrm{N}_{2} \mathrm{H}^{+}$and $\mathrm{N}_{2} \mathrm{D}^{+}$.

In this paper, we first introduce a more complete chemical network and describe our methods of following chemical evolution $(\$ 2$. Then in $\$ 3$ we present our results for determining the chemical age of cloud cores by the deuterium fraction of $\mathrm{N}_{2} \mathrm{H}^{+}$. This is similar to the approaches of P09 and Pagani et al. (2013), but extended to cover a broader range of conditions, including those relevant to high-mass star-forming regions that may contain massive starless cores (e.g., Tan et al. 2013). Furthermore, we consider a range of simple parameterized collapse rates relative to that of free-fall collapse. The implications of our results are discussed in \$4 including detailed comparison with the results of P09 and Pagani et al. 2013). Conclusions are summarized in $\$ 5$

\section{METHODS}

\subsection{Fiducial Chemical Network}

Our model is based on the network first described in Vastel et al. (2012) (hereafter V12), who used the chemistry results to interpret ground-based and Herschel Space Observatory observations of deuterated isotopologues of $\mathrm{H}_{3}^{+}$toward a pre-stellar core (see description in their §3.3). The V12 code was originally built starting from a complete reaction network including only molecules with up to three atoms in size, extracted from the Nahoon network (Wakelam et al. 2012), which is available in the KIDA database (Oct. 2010 version).

\footnotetext{
${ }^{1}$ http://kida.obs.u-bordeaux1.fr/
}

The reduced network only includes the elements $\mathrm{H}, \mathrm{D}$, $\mathrm{He}, \mathrm{O}, \mathrm{C}$ and $\mathrm{N}$. This simplified network still allows us to follow easy-to-observe species in the gas phase, such as $\mathrm{N}_{2} \mathrm{H}^{+}, \mathrm{HCO}^{+}$and their deuterated forms. The reduced network includes the spin states of $\mathrm{H}_{2}, \mathrm{H}_{3}^{+}$and their deuterated isotopologues, following prescriptions of Walmsley et al. (2004), Flower et al. (2006), Hugo et al. (2009), P09, Sipilä et al. (2010), and selecting the most recent values for the rate coefficients from the 2010 KIDA database.

We have made five main improvements to the V12 network: (1) The dissociative recombination rates of all the forms of $\mathrm{H}_{3}^{+}$have been calculated through the interpolation of Table B.1 of P09. (2) Rate coefficients have been updated and recombination reactions of $\mathrm{C}^{+}, \mathrm{N}^{+}$and $\mathrm{O}^{+}$ onto negatively charged dust grains have been added, following the more recent 2011 KIDA network. (3) Bugs in the duplication routine used to construct the V12 network have been corrected. In particular, we adjusted the branching ratio of reactions such as

$$
\begin{aligned}
& \mathrm{HD}+\mathrm{CO}^{+} \rightarrow \mathrm{H}+\mathrm{DCO}^{+} \\
& \mathrm{HD}+\mathrm{CO}^{+} \rightarrow \mathrm{D}+\mathrm{HCO}^{+},
\end{aligned}
$$

which is now a half of that of the following reaction from KIDA:

$$
\mathrm{H}_{2}+\mathrm{CO}^{+} \rightarrow \mathrm{H}+\mathrm{HCO}^{+} \text {. }
$$

(4) We checked our network against that of Sipilä et al. (2013) (hereafter S13) to make sure that spin-state rules were followed. This implied the elimination of some reactions, such as charge exchange reactions involving spin changes; the elimination of reactions of the type

$$
\mathrm{H}_{2} \mathrm{O}+\mathrm{H}^{-} \rightarrow \mathrm{O}-\mathrm{H}_{2}+\mathrm{OH}^{-}
$$

as in cold gas it is assumed that only $\mathrm{p}-\mathrm{H}_{2}$ can form in reactions containing only reactants other than $\mathrm{H}_{2}^{+}, \mathrm{H}_{2}$ and $\mathrm{H}_{3}^{+}$; the elimination of a few reactions built by the V12 duplication code which did not follow Oka (2004) spin rules, such as

$$
\mathrm{p}-\mathrm{H}_{2}+\mathrm{p}-\mathrm{D}_{2}^{+} \rightarrow \mathrm{H}+\mathrm{O}-\mathrm{D}_{2} \mathrm{H}^{+} .
$$

(5) Because of its importance for Oxygen chemistry, we include $\mathrm{H}_{3} \mathrm{O}^{+}$and its deuterated isotopologues, as well as all reactions involving species that are present in our network. The inclusion of $\mathrm{H}_{3} \mathrm{O}^{+}$significantly improves the overall agreement with $\mathrm{S} 13$. The abundances of electrons, water, $\mathrm{CO}, \mathrm{HCO}^{+}, \mathrm{DCO}^{+}, \mathrm{N}_{2}, \mathrm{~N}_{2} \mathrm{H}^{+}, \mathrm{N}_{2} \mathrm{D}^{+}$(the most important species in the network, as the deuterium fraction is typically measured through the $\mathrm{N}_{2} \mathrm{D}^{+} / \mathrm{N}_{2} \mathrm{H}^{+}$ and $/$ or $\mathrm{DCO}^{+} / \mathrm{HCO}^{+}$column density ratios) are always within a factor of 2 when compared to S13 network. This is also true for deuterium fraction and its equilibrium timescale (defined and studied later in \$3.2). We did not include the surface chemistry described in S13, since there are large uncertainties involved, while not significantly impacting the gas-phase chemistry of $\mathrm{H}_{3}^{+}, \mathrm{HCO}^{+}$, $\mathrm{N}_{2} \mathrm{H}^{+}$and their deuterated forms in cold regions (see 3.5 . However, the surface formation of $\mathrm{p}-\mathrm{H}_{2}, \mathrm{o}-\mathrm{H}_{2}, \mathrm{HD}$, $\mathrm{p}-\mathrm{D}_{2}$ and $\mathrm{o}-\mathrm{D}_{2}$ are included in our network. The rates have been calculated following Le Petit et al. (2002). The ortho-to-para ratio upon surface formation has been assumed equal to the statistical value of 3 for $\mathrm{H}_{2}$ and 2 
for $\mathrm{D}_{2}$. Neutral and negatively charged grains are considered. Coulomb focusing was taken into account for reactions involving positively charged ions on negatively charged grains (Draine \& Sutin 1987). Our fiducial chemical network now includes 3232 reactions involving 132 different species. The network will be publicly available via the KIDA database.

Our first treatment of molecular freeze-out involves an approximation of reducing the initial elemental abundances of species heavier than He by a "depletion factor", $f_{\mathrm{D}}$ (=10 for the fiducial model, fixed in each run). Below, we also describe an extension of this simple approximation to include time-dependent depletion and desorption $(2.2)$.

\subsection{Time-Dependent Depletion/Desorption}

The inclusion of time-dependent depletion and desorption rates of the heavier elements adds additional uncertainty and complexity to the modeling (which is why in the fiducial network, above, depletion factor is treated as a controllable parameter). However, in order to gain a basic insight into the potential effects of these more complex processes, we developed a second network that includes freeze-out and desorption of neutral species, following Hasegawa et al. (1992) and Hasegawa \& Herbst (1993). Hereafter we refer to this as the Time-Dependent Depletion (TDD) network. Three types of reactions are implemented: (a) sticking onto dust grains; (b) thermal evaporation; (c) CR induced evaporation. Binding energies and sticking coefficients are the same as those used in Garrod et al. (2007). Altogether there are 153 new reactions added into the TDD network.

\subsection{Fiducial Initial Conditions and Model Parameters}

We choose the following fiducial initial conditions and model parameters (see also Table 1). The density is expressed via the number density of $\mathrm{H}$ nuclei, $n_{\mathrm{H}}=$ $10^{5} \mathrm{~cm}^{-3}$, gas temperature, $T=15 \mathrm{~K}$ and heavy element depletion factor, $f_{\mathrm{D}}=10$. The choices of these fiducial values are motivated by observations of both low- and high-mass pre-stellar cores (e.g. Ward-Thompson et al. 1999, Crapsi et al. 2005, 2007; Pillai et al. 2006; Hernandez et al. 2011; Ragan et al. |2011; Butler \& Tan 2012). The CR ionization rate, $\zeta=2.5 \times 10^{-17} \mathrm{~s}^{-1}$, is adopted from van der Tak \& van Dishoeck (2000).

The fiducial visual extinction, $A_{V}$, is set to $30 \mathrm{mag}$, a value large enough so that photochemistry is unimportant for our adopted radiation field (standard Habing field, $\left.G_{0}=1\right)$. We assume that refractory metals of low ionization potential (such as $\mathrm{Mg}$ and $\mathrm{Fe}$ ) and polycyclic aromatic hydrocarbons (PAHs), important for the ionization structure, are not present in gas phase because of freeze-out onto dust grains, a reasonable assumption in cold molecular clouds (see Caselli et al.|1998; Wakelam \& Herbst 2008, for the effects of metals and PAHs, respectively, on the chemical structure of molecular clouds). The dust-to-gas mass ratio, grain radius and grain density are taken from the original Nahoon model and represent the fiducial values typically adopted in chemical models.

The fiducial initial fractional abundances of elements, with respect to total $\mathrm{H}$ nuclei are listed in Table 2 For simplicity, all species are assumed to be in atomic form,
Table 1

Fiducial Parameter Values.

\begin{tabular}{ccc}
\hline \hline Parameter & Description & Fiducial value \\
\hline$n_{\mathrm{H}}$ & number density of H nuclei & $1.0 \times 10^{5} \mathrm{~cm}^{-3}$ \\
$T$ & temperature & $15 \mathrm{~K}$ \\
$\zeta$ & CR ionization rate & $2.5 \times 10^{-17} \mathrm{~s}^{-1}$ \\
$f_{\mathrm{D}}$ & depletion factor & 10 \\
$G_{0}$ & ratio to Habing field & 1 \\
$A_{V}$ & visual extinction & $30 \mathrm{mag}$ \\
$\mathrm{DGR}^{\mathrm{a}}$ & dust-to-gas mass ratio & $7.09 \times 10^{-3}$ \\
$a_{0}$ & dust particle radius & $1.0 \times 10^{-5} \mathrm{~cm}$ \\
$\rho_{\text {GRAIN }}$ & dust grain density & $3.0 \mathrm{~g} \mathrm{~cm}^{-3}$ \\
& &
\end{tabular}

a Following Draine 2011.

Table 2

Fiducial initial elemental abundances.

\begin{tabular}{cc}
\hline \hline Species & Abundance $\left(n_{\text {species }} / n_{\mathrm{H}}\right)$ \\
\hline $\mathrm{p}-\mathrm{H}_{2}$ & $1.25 \times 10^{-01}$ \\
$\mathrm{O}-\mathrm{H}_{2}$ & $3.75 \times 10^{-01}$ \\
$\mathrm{HD}$ & $1.50 \times 10^{-05}$ \\
$\mathrm{He}$ & $1.00 \times 10^{-01}$ \\
$\mathrm{~N}$ & $2.10 \times 10^{-06}$ \\
$\mathrm{O}$ & $1.80 \times 10^{-05}$ \\
$\mathrm{C}$ & $7.30 \times 10^{-06}$ \\
GRAIN0 & $1.32 \times 10^{-12}$ \\
\hline
\end{tabular}

except for $\mathrm{H}$ and $\mathrm{D}$. Deuterium is initially assumed to be all in HD, with a fractional abundance adopted from the measurement of the elemental $[\mathrm{D}] /[\mathrm{H}]$ ratio measured in the Galactic interstellar medium $\left([\mathrm{D}] /[\mathrm{H}] \sim 1.5 \times 10^{-5}\right.$; e.g. Oliveira et al. 2003). Below, we also investigate the effects of changing these initial chemical states, finding that our main results are quite insensitive to these choices.

The fiducial initial ortho-to-para $\mathrm{H}_{2}$ ratio, $\mathrm{OPR}^{\mathrm{H}_{2}}$, is set to its statistical value of 3 , assumed to be obtained in the process of $\mathrm{H}_{2}$ formation on dust grain surfaces. This choice does impact deuterium chemistry, and so below we do consider the effects of a range of initial values.

\section{RESULTS}

\subsection{The Fiducial Model}

Figure 1 shows the fractional abundances ([species]= $n_{\text {species }} / n_{\mathrm{H}}$ ) of important species as a function of time in the fiducial model, i.e. the fiducial network with fiducial initial conditions. As the gas evolves under these cold, dense conditions, the deuteration becomes active through the exothermic reaction (only true with respect to para states of reactants and products; Pagani et al. 1992):

$$
\mathrm{p}-\mathrm{H}_{3}^{+}+\mathrm{HD} \rightleftharpoons \mathrm{p}-\mathrm{H}_{2} \mathrm{D}^{+}+\mathrm{p}-\mathrm{H}_{2}+232 \mathrm{~K} .
$$

$\mathrm{H}_{2} \mathrm{D}^{+}$can cede a deuteron to major neutral species, such as $\mathrm{CO}$ and $\mathrm{N}_{2}$, producing $\mathrm{DCO}^{+}$and $\mathrm{N}_{2} \mathrm{D}^{+}$, respectively. As a consequence, the deuterium fraction (i.e., defined by the abundance ratios $\left[\mathrm{N}_{2} \mathrm{D}^{+}\right] /\left[\mathrm{N}_{2} \mathrm{H}^{+}\right]$, $\left.\left[\mathrm{DCO}^{+}\right] /\left[\mathrm{HCO}^{+}\right]\right)$starts to overcome the cosmic abundance of deuterium. Hereafter, we denote the deuterium fraction of a certain species as $D_{\text {frac }}^{\text {species }}$ (e.g. $\left.\left[\mathrm{N}_{2} \mathrm{D}^{+}\right] /\left[\mathrm{N}_{2} \mathrm{H}^{+}\right] \equiv D_{\text {frac }}^{\mathrm{N}_{2} \mathrm{H}^{+}}\right)$and the spin-state ratio as $\mathrm{OPR}^{\text {species }}$ (e.g., $\left[\mathrm{o}-\mathrm{H}_{2}\right] /\left[\mathrm{p}-\mathrm{H}_{2}\right] \equiv \mathrm{OPR}^{\mathrm{H}_{2}}$ ). We will focus 


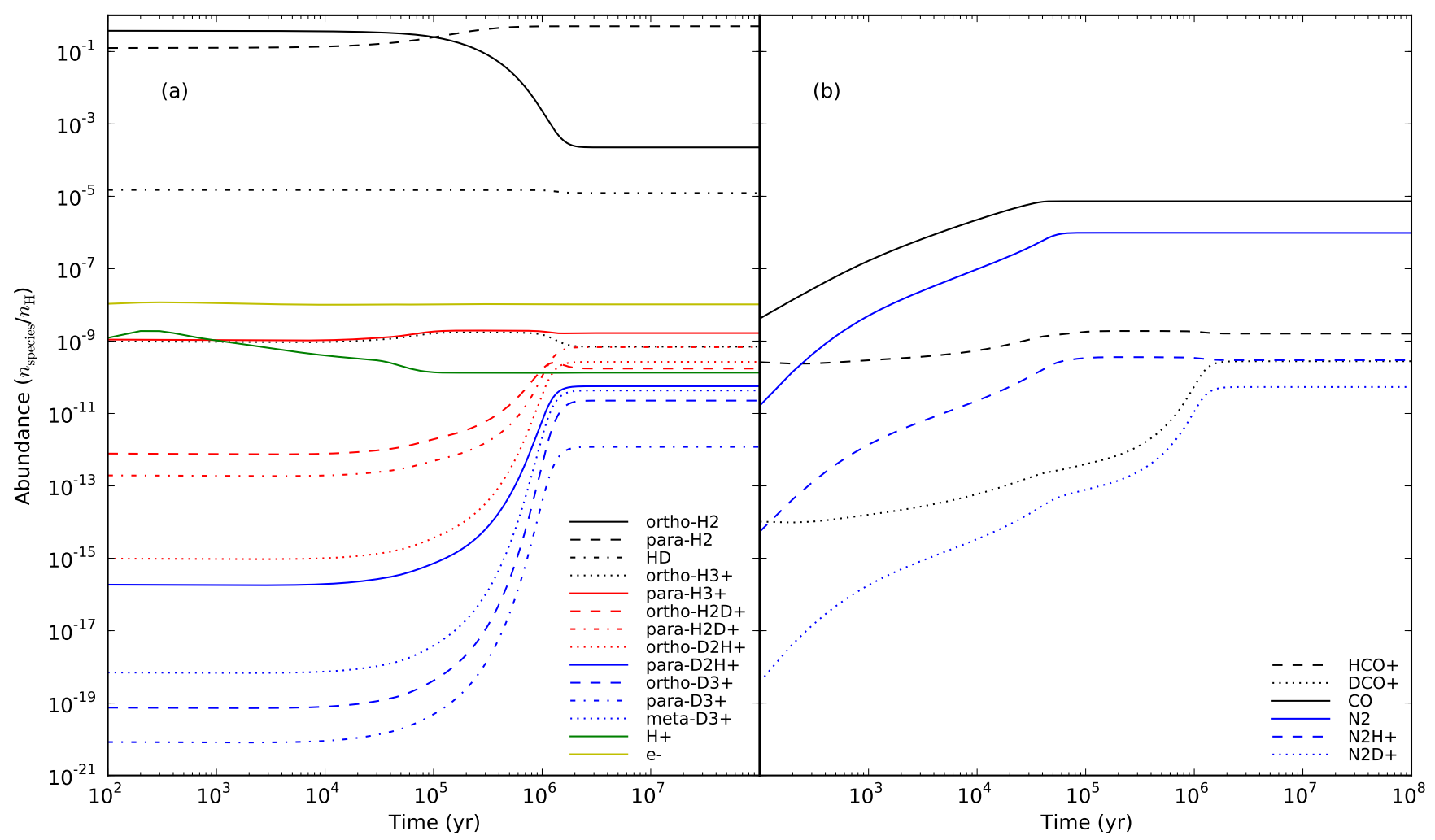

Figure 1. Time evolution of fractional abundances of important species in the fiducial model with $n_{\mathrm{H}}=1.0 \times 10^{5} \mathrm{~cm}^{-3}, T=15 \mathrm{~K}$, $\zeta=2.5 \times 10^{-17} \mathrm{~s}^{-1}, f_{\mathrm{D}}=10, G_{0}=1$ and $A_{V}=30 \mathrm{mag}$. (a): hydrogen species, including $\mathrm{H}_{2}, \mathrm{H}_{3}^{+}$and their deuterated isotopologues (plus spin states), and electrons. (b): Species of our interest, especially $\mathrm{N}_{2} \mathrm{D}^{+}, \mathrm{N}_{2} \mathrm{H}^{+}$, and their progenitor $\mathrm{N}_{2}$.

on $D_{\text {frac }}^{\mathrm{N}_{2} \mathrm{H}^{+}}$in our study, since $\mathrm{HCO}^{+}$suffers more from depletion than $\mathrm{N}_{2} \mathrm{H}^{+}$, so that $D_{\text {frac }}^{\mathrm{N}_{2} \mathrm{H}^{+}}$is a better tool for tracing the inner, denser regions of starless/pre-stellar cores (Caselli et al. 2002, Crapsi et al. 2005).

The deuterium fraction, shown in Figure 2, increases significantly only at times later than $\sim 10^{5} \mathrm{yr}$, when the abundance of $\mathrm{o}_{2} \mathrm{H}_{2}$ starts to drop. Deuteration is suppressed by $\mathrm{o}-\mathrm{H}_{2}$, which drives the reaction (6) backwards, as originally pointed out by Pineau des Forets et al. (1991) (for $\mathrm{p}-\mathrm{H}_{2} \mathrm{D}^{+}$) and Pagani et al. (1992) (for $\left.\mathrm{O}^{-} \mathrm{H}_{2} \mathrm{D}^{+}\right)$, and later discussed by Flower et al. (2006), P09, and Pagani et al. (2011). The conversion of $\mathrm{O}^{-}$ $\mathrm{H}_{2}$ to $\mathrm{p}-\mathrm{H}_{2}$ mainly proceeds through the reactions of $\mathrm{O}_{-} \mathrm{H}_{2}$ with $\mathrm{H}^{+}$and $\mathrm{H}_{3}^{+}$. Figure 2 shows how $\mathrm{OPR}^{\mathrm{H}_{2}}$ and $D_{\text {frac }}^{\mathrm{N}_{2} \mathrm{H}^{+}}$change together in the fiducial model: as expected, $D_{\text {frac }}^{\mathrm{N}_{2} \mathrm{H}^{+}}$goes up as $\mathrm{OPR}^{\mathrm{H}_{2}}$ drops. After reaching the equilibrium steady-state at about 2 million years, $D_{\text {frac }}^{\mathrm{N}_{2} \mathrm{H}^{+}}$has increased by about 4 orders of magnitude relative to the cosmic deuterium to hydrogen abundance ratio, while $\mathrm{OPR}^{\mathrm{H}_{2}}$ has dropped by more than 3 orders of magnitude. One can also see from Figure 1 that all species reach steady-state when $\mathrm{OPR}^{\mathrm{H}_{2}}$ does. These results emphasize that $\mathrm{OPR}^{\mathrm{H}_{2}}$ is crucial for cold gas chemistry in general, for deuterium fractionation in particular and for the chemical timescale (see $\$ 3.3 .2$ ).

One thing to note is that in our model the abundance of heavy molecules such as $\mathrm{CO}$ and $\mathrm{N}_{2}$ increase with time (Fig.11). As $\mathrm{CO}$ and $\mathrm{N}_{2}$ are both important destruction partners of $\mathrm{H}_{3}^{+}$and its deuterated isotopologues, their increasing abundance would tend to reduce that of these

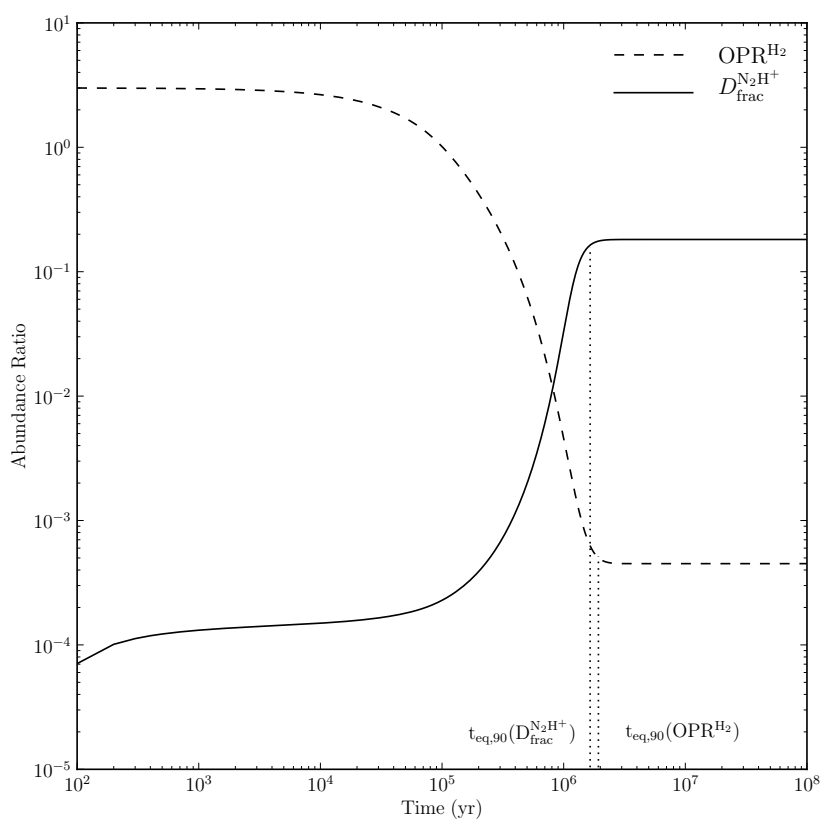

Figure 2. Time evolution of $\mathrm{OPR}^{\mathrm{H}_{2}} \equiv\left[\mathrm{o}-\mathrm{H}_{2}\right] /\left[\mathrm{p}-\mathrm{H}_{2}\right]$ and $D_{\text {frac }}^{\mathrm{N}_{2} \mathrm{H}^{+}} \equiv\left[\mathrm{N}_{2} \mathrm{D}^{+}\right] /\left[\mathrm{N}_{2} \mathrm{H}^{+}\right]$in the fiducial model. Two dotted lines mark the times when these quantities approach within $10 \%$ of their final equilibrium values $\left(t_{\mathrm{eq}, 90}\right)$.

species. However, the countervailing effect of the decreasing abundance of $\mathrm{o}-\mathrm{H}_{2}$ is more dominant. At the physical conditions of the fiducial model, as time proceeds, species like CO should suffer from increasing amounts of freeze- 
out onto dust grains (e.g. Caselli et al. 1999). We have not included any differential treeze-out mechanism for $\mathrm{CO}$ and $\mathrm{N}_{2}$, as laboratory work has found similar sticking coefficients and binding energies for the two molecules (Bisschop et al. 2006). Recall also that this fiducial network assumes a fixed, heavy element-independent depletion factor is present from the initial condition. The effects of relaxing this assumption are investigated in \$3.5.

With these caveats in mind, we note from Figure 1 that the $\mathrm{N}_{2} \mathrm{H}^{+} / \mathrm{CO}$ ratio increases with time, up to a few times $10^{5} \mathrm{yr}$, when the $\mathrm{N}_{2} \mathrm{H}^{+}$abundance reaches steady state, as $\mathrm{N}_{2}$, the precursor molecule to $\mathrm{N}_{2} \mathrm{H}^{+}$, forms more slowly than CO, via neutral-neutral reactions rather than ion-neutral reactions (see also Hily-Blant et al. 2010).

\subsection{The Deuteration Timescale}

Studies have suggested a theoretical relation between the deuterium fraction and the evolutionary stage in lowmass cores (Caselli 2002, Crapsi et al. 2005, Pagani et al. 2013 ), with the level of deuteration rising with increasing age and density of the starless core, before then falling once a protostar forms and starts to heat its natal envelope. Fontani et al. (2011) have examined a similar relation in massive cores, and their findings support the use of deuterium fraction as an evolutionary indicator for massive starless and star-forming cores.

Here we investigate the absolute timescale for the growth of the deuterium fraction and its implication for the ages of low-mass and massive starless cores. We also examine how the variation of physical properties of the gas, including choices of initial conditions, influences this deuteration timescale, i.e. a chemical timescale. For convenience, when considering the output of our chemical network, we define the equilibrium deuterium fraction, $D_{\text {frac,eq }}$ as the average of two adjacent outputs of $D_{\text {frac }}$ (separated by $\Delta t=10^{4} \mathrm{yr}$ ) that have a fractional change

$$
\left|\Delta D_{\text {frac }}\right| / D_{\text {frac }}<\epsilon,
$$

with a choice of $\epsilon=5 \times 10^{-5}$. In practice, we run the model for $10^{8} \mathrm{yr}$ and then search backwards in time for when this condition is satisfied. We denote the timescale to reach the equilibrium condition defined by Eq. (7) as $t_{\text {eq }}\left(D_{\text {frac }}^{\text {species }}\right)$. The equilibrium value of the ortho-to-para ratio of $\mathrm{H}_{2}, \mathrm{OPR}_{\mathrm{eq}}^{\mathrm{H}_{2}}$, is defined in a similar way, and the timescale is denoted $t_{\text {eq }}\left(\mathrm{OPR}^{\mathrm{H}_{2}}\right)$. In practice, since the evolution of $D_{\text {frac }}^{\mathrm{N}_{2} \mathrm{H}^{+}}$and $\mathrm{OPR}_{\text {eq }}^{\mathrm{H}_{2}}$ are very slow as they approach equilibrium (e.g. Fig. 2), we also define a more representative equilibrium timescale $t_{\mathrm{eq}, 90}\left(D_{\mathrm{frac}}^{\mathrm{N}_{2} \mathrm{H}^{+}}\right)$ as the time when $D_{\text {frac }}^{\mathrm{N}_{2} \mathrm{H}^{+}}$increases to $90 \%$ of $D_{\text {frac,eq }}^{\mathrm{N}_{2} \mathrm{H}^{+}}$. In a similar way, we define $t_{\mathrm{eq}, 90}\left(\mathrm{OPR}^{\mathrm{H}_{2}}\right)$ as the time when $\mathrm{OPR}^{\mathrm{H}_{2}}$ decreases to $\mathrm{OPR}_{\text {eq }}^{\mathrm{H}_{2}} / 0.90$.

We will compare these chemical timescales to physical timescales, in particular the local free-fall timescale, $t_{\mathrm{ff}}$, which, for a uniform density core, is

$$
t_{\mathrm{ff}}=\left(\frac{3 \pi}{32 G \rho}\right)^{1 / 2}=1.39 \times 10^{5}\left(\frac{n_{\mathrm{H}}}{10^{5} \mathrm{~cm}^{-3}}\right)^{-1 / 2} \mathrm{yr} .
$$

Note that this timescale is evaluated with reference to the current density of a core, predicting how long it will take in the future to collapse to a very high density state in the absence of any internal pressure support. However, this timescale is also an approximate estimate for the minimum amount of time that the core has existed at densities similar to its current value, since if contraction is driven by self-gravity we do not expect evolution in core properties to be proceeding on timescales shorter than the local free-fall time.

Furthermore, depending on the degree of turbulent and magnetic field support, the contraction could be proceeding at rates much slower than that of free-fall collapse. Thus, in \$3.6. we will also consider models in which the density evolves continuously at various rates relative to the local free-fall time.

Note that the deuteration timescale refers to the age of a core, which in our fiducial modelling is the time spent at the given constant density. In the evolving density models, the deuteration timescale is the time the core has spent evolving from a particular lower density initial condition to the current density. When comparing to observations, one has to take into account that dense cores have been evolving from lower densities, so models with evolving density structures are important to constrain the rates of collapse from the measured abundances of deuterated molecules.

The first line of Table 3 lists equilibrium abundance ratios and timescales for the fiducial model, i.e. with $n_{\mathrm{H}}$ $=10^{5} \mathrm{~cm}^{-3}$. The deuteration timescale $t_{\mathrm{eq}, 90}\left(D_{\mathrm{frac}}^{\mathrm{N}_{2} \mathrm{H}^{+}}\right)$is $\simeq 12 t_{\mathrm{ff}}$. Thus, if a starless core were to be observed with physical and environmental properties equal to the fiducial model, and $D_{\text {frac }} \gtrsim 0.1$, then our modeling implies it would need to be substantially older than its current local $t_{\mathrm{ff}}$, assuming it had started with our adopted initial conditions, including the initial $\mathrm{OPR}$ of $\mathrm{H}_{2}$.

\subsection{Effect of Initial Conditions on the Deuteration Timescale}

\subsubsection{Initial Elemental Abundances}

As shown in Table 2, the fiducial model starts with $\mathrm{H}$ in molecular form, D in $\mathrm{HD}$, while $\mathrm{He}, \mathrm{C}, \mathrm{N}, \mathrm{O}$ are in atomic form. However, when dense cores form in molecular clouds, a large fraction of $\mathrm{CO}$ and maybe $\mathrm{N}_{2}$ should already be present (see also $\mathrm{Li}$ et al. 2013). There is some evidence that a significant fraction of the nitrogen is still in atomic form in dense cores due to the slow conversion from $\mathrm{N}$ to $\mathrm{N}_{2}$, but the exact amount is unclear (Hily-Blant et al. 2010). As different initial abundances could impact $D_{\text {frac,eq }}^{\text {species }}$ and $t_{\text {eq }, 90}\left(D_{\text {frac }}^{\text {species }}\right)$, we quantify these effects considering 3 variations to the fiducial model descibed in \$2.3 (1) "atomic D", where D is in atomic form, compared to the fiducial model, assuming that $\mathrm{H}_{2}$ is in molecular form; (2) "fully molecular", where everything starts in molecular form (all $\mathrm{N}$ in $\mathrm{N}_{2}$, all $\mathrm{C}$ in $\mathrm{CO}$, with the leftover Oxygen left in atomic form); (3) "half $\mathrm{N}$ in $\mathrm{N}_{2}$ ", where half of the Nitrogen is left in atomic form compared to "fully molecular" case.

Figure 3 shows the results of these tests, focussing on the effects on the time evolution of $D_{\text {frac }}^{\mathrm{N}_{2} \mathrm{H}^{+}}$and $\mathrm{OPR}^{\mathrm{H}_{2}}$. Table 3 lists the equilibrium ratios and timescales. The choice of initial atomic versus molecular abundances has no effect on the equilibrium abundance ratios and has little effect $(\lesssim 1 \%)$ on the timescales. 
Table 3

Equilibrium abundance ratios and timescales for models with various initial conditions.

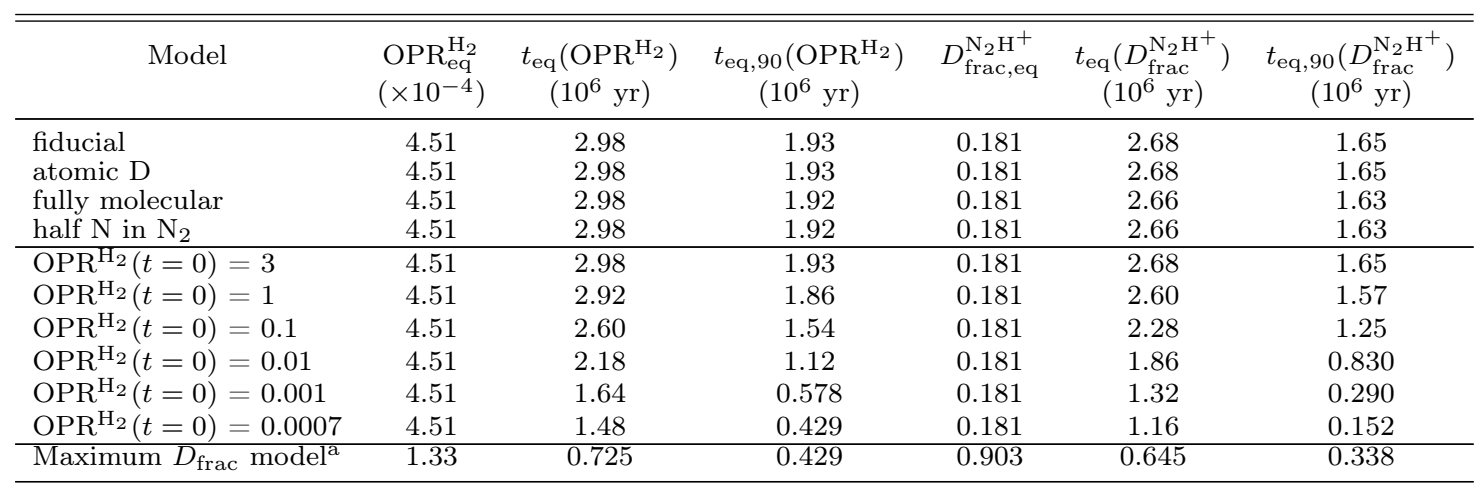

a See 3.4 .6

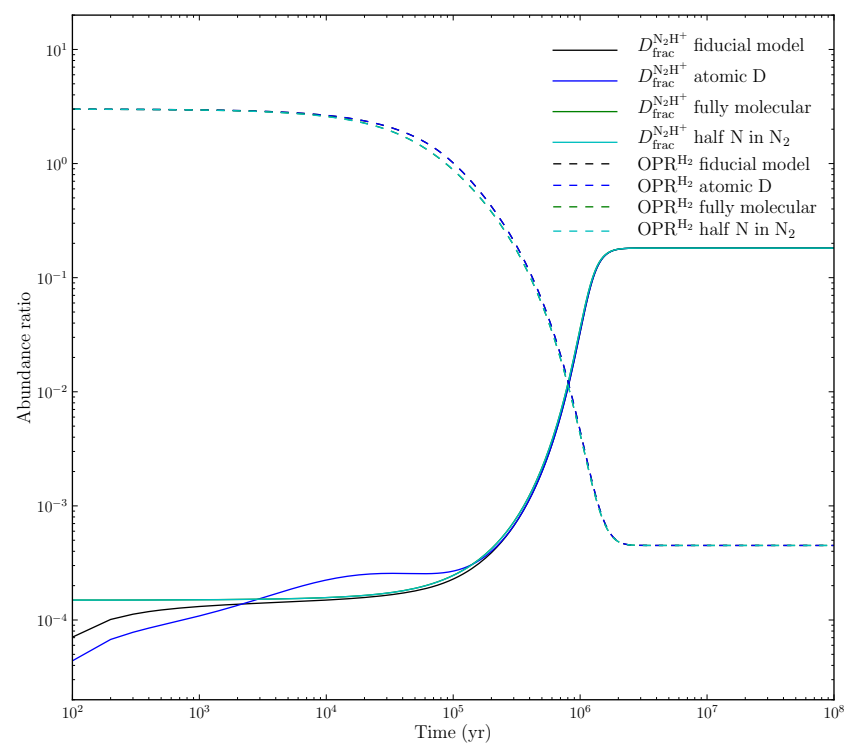

Figure 3. Time evolution of $\mathrm{OPR}^{\mathrm{H}_{2}}$ and $D_{\text {frac }}^{\mathrm{N}_{2} \mathrm{H}^{+}}$with 4 sets of initial elemental abundances. See 3.3 .1 for the description of these sets. The equilibrium ratios and timescales are summarized in Table 3.

\subsubsection{Initial $O P R^{\mathrm{H}_{2}}$}

Another poorly constrained, but crucial, parameter is the initial $\mathrm{OPR}^{\mathrm{H}_{2}}$. There are only a few studies yielding observational constraints: in diffuse clouds, Crabtree et al. (2011) measured $\mathrm{OPR}^{\mathrm{H}_{2}} \simeq 0.3-0.8$; in the prestellar core L183, P09 derived $\mathrm{OPR}^{\mathrm{H}_{2}} \simeq 0.1$ (see also Pagani et al. 2011), while Troscompt et al. (2009) estimated $\mathrm{OPR}^{\mathrm{H}_{2}}<1$ and Maret \& Bergin (2007) estimated $\mathrm{OPR}^{\mathrm{H}_{2}} \sim 0.015$ in the starless Bok globule B68 (see also discussion in Flower et al. 2006, S13). Evidently, different environmental conditions strongly impact $\mathrm{OPR}^{\mathrm{H}_{2}}$ (as also deduced by Caselli et al. 2008, in their study of $\mathrm{o}-\mathrm{H}_{2} \mathrm{D}^{+}$in star-forming regions).

Our fiducial model starts with $\mathrm{OPR}^{\mathrm{H}_{2}}=3$, which implies that all $\mathrm{H}_{2}$ molecules are initially in their statistical spin ratio, as expected if they have just been formed on the surface of dust grains, i.e. if the molecular cloud is very young. However, this may not be the case, based on the above mentioned observations in diffuse clouds and if cloud cores form at a later stage compared to the formation of the parent molecular cloud. To explore this, we consider the effect of different initial $\mathrm{OPR}^{\mathrm{H}_{2}}$ values in the fiducial model. Fig. 4 shows their effects on the time evolution of $\mathrm{OPR}^{\mathrm{H}_{2}}$ and $D_{\text {frac }}^{\mathrm{N}_{2} \mathrm{H}^{+}}$. The different initial $\mathrm{OPR}^{\mathrm{H}_{2}}$ values have little effect on both $\mathrm{OPR}_{\text {eq }}^{\mathrm{H}_{2}}$ and $D_{\text {frac,eq }}^{\mathrm{N}_{2} \mathrm{H}^{+}}$, but the timescales to reach equilibrium are changed (as also found by Pagani et al. 2011, see their Fig. 2). Since the $\mathrm{OPR}_{\mathrm{eq}}^{\mathrm{H}_{2}}$ is $4.51 \times 10^{-4}$, the lower the initial $\mathrm{OPR}^{\mathrm{H}_{2}}$, the sooner chemical equilibrium will be reached. We find $t_{\mathrm{eq}, 90}\left(D_{\mathrm{frac}}^{\mathrm{N}_{2} \mathrm{H}^{+}}\right)$becomes similar to $t_{\mathrm{ff}}$ if $\mathrm{OPR}^{\mathrm{H}_{2}}$ is initially 0.001 or lower. This is also summarized in Table 3 . These results suggest that we should in general consider the possible effects of starting with much lower values of the initial $\mathrm{OPR}^{\mathrm{H}_{2}}$ than the fiducial value of 3 (although initial values lower than 0.1 are not consistent with $\mathrm{DCO}^{+}$observations, as discussed in Pagani et al. 2011).

\subsection{Effect of Starless Core Physical and Environmental Properties on the Deuteration Timescale}

Here we present a parameter space exploration to see how different physical conditions impact the chemical evolution of gas in starless cores. We will first assume an initial $\mathrm{OPR}^{\mathrm{H}_{2}}=3$ (results for $\mathrm{OPR}^{\mathrm{H}_{2}}=1,0.1,0.01$ are discussed below in 3.4.5. Then, we vary four parameters: the $\mathrm{H}$ number density $n_{\mathrm{H}}$ from $10^{3}$ to $10^{7} \mathrm{~cm}^{-3}$, the temperature $T$ from 5 to $30 \mathrm{~K}$, the $\mathrm{CR}$ ionization rate $\zeta$ from $10^{-18}$ to $10^{-15} \mathrm{~s}^{-1}$, the gas phase depletion factor $f_{\mathrm{D}}$ from 1 to $1000\left(f_{\mathrm{D}}=1 \text { implies no depletion }\right)^{2}$. These ranges of parameter space are chosen to cover conditions expected for both low and high-mass starless cores (e.g., Bergin \& Tafalla 2007; Tan et al. 2014).

Figure 5 shows the effect on the time evolution of $\mathrm{OPR}^{\mathrm{H}_{2}}$ and $D_{\text {frac }}^{\mathrm{N}_{2} \mathrm{H}^{+}}$of varying these four parameters. In general, $n_{\mathrm{H}}$ and $T$ have a greater influence on $D_{\text {frac,eq }}^{\mathrm{N}_{2} \mathrm{H}^{+}}$, while $\zeta$ and $f_{\mathrm{D}}$ has an effect on both $D_{\text {frac,eq }}^{\mathrm{N}_{2} \mathrm{H}^{+}}$and $\mathrm{OPR}^{\mathrm{H}_{2}}$.

\footnotetext{
${ }^{2}$ Note that some small regions of parameter space are not internally self-consistent, e.g. a very cold temperature model with very high CR ionization rate, but our goal here is to first explore the effects of each variable on the deuteration chemistry in isolation, before later building self-consistent thermodynamic models.
} 


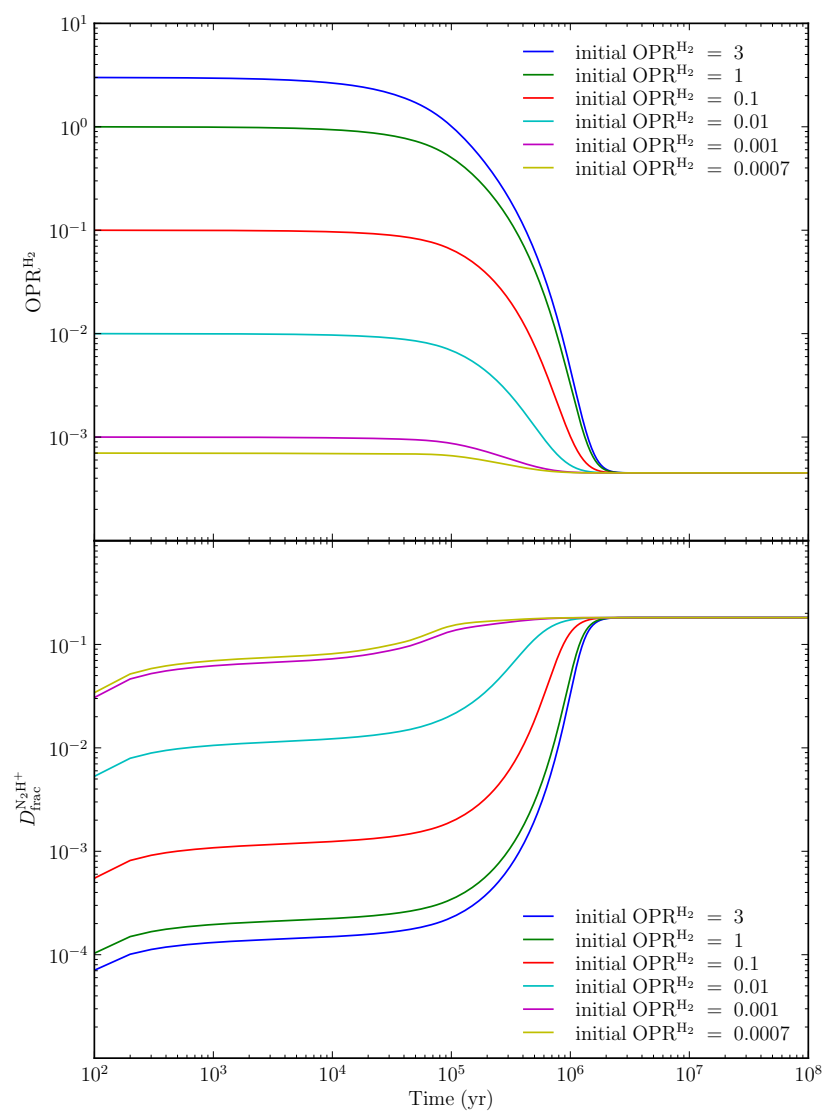

Figure 4. Time evolution of $\mathrm{OPR}^{\mathrm{H}_{2}}$ (top) and $D_{\text {frac }}^{\mathrm{N}_{2} \mathrm{H}^{+}}$(bottom) under different assumptions of initial $\mathrm{OPR}^{\mathrm{H}_{2}}$. We explore initial $\mathrm{OPR}^{\mathrm{H}_{2}}$ from 3 (the fiducial model) down to $7 \times 10^{-4}$. Note that $\mathrm{OPR}_{\mathrm{eq}}^{\mathrm{H}_{2}} \simeq 4.51 \times 10^{-4}$.

This implies that the physical environment plays an important role in dense core chemistry. It is thus crucial to have good observational constraints on these properties when trying to model observed cores.

Figure 6 shows the variation of the equilibrium ratios and timescales of $\mathrm{OPR}^{\mathrm{H}_{2}}$ (upper 2 rows) and $D_{\mathrm{frac}}^{\mathrm{N}_{2} \mathrm{H}^{+}}$ (lower 2 rows). Note that the variation of the equilibrium time of $D_{\text {frac }}^{\mathrm{N}_{2} \mathrm{H}^{+}}$(fourth row) is very similar to that of $\mathrm{OPR}^{\mathrm{H}_{2}}$ (second row), as explained in \$3.1. In the following, we summarize their dependence on each physical quantity, with emphasis on $D_{\text {frac }}^{\mathrm{N}_{2} \mathrm{H}^{+}}$and $t_{\text {eq, } 90}\left(D_{\text {frac }}^{\mathrm{N}_{2} \mathrm{H}^{+}}\right)$.

\subsubsection{Dependence on $n_{\mathrm{H}}$}

As shown in panel (i) of Figure 6, a denser core will have a higher $D_{\text {frac,eq }}^{\mathrm{N}_{2} \mathrm{H}^{+}}$. The $D_{\text {frac,eq }}^{\mathrm{N}_{2} \mathrm{H}^{+}}$changes by $\sim$ an order of magnitude, from $3.21 \times 10^{-2}$ at $n_{\mathrm{H}}=10^{3} \mathrm{~cm}^{-3}$, to $3.51 \times 10^{-1}$ at $n_{\mathrm{H}}=10^{7} \mathrm{~cm}^{-3}$.

From panel $(\mathrm{m})$ we see that $t_{\mathrm{eq}, 90}\left(D_{\text {frac }}^{\mathrm{N}_{2} \mathrm{H}^{+}}\right)$varies with $n_{\mathrm{H}}$ by a factor of $\sim 7$. Thus, cores with a wide range of densities have similar deuteration timescales, if other conditions are fixed. When $n_{\mathrm{H}} \gtrsim 3 \times 10^{4} \mathrm{~cm}^{-3}$, $t_{\text {eq,90 }}\left(D_{\text {frac }}^{\mathrm{N}_{2} \mathrm{H}^{+}}\right)$and $t_{\text {eq,90 }}\left(\mathrm{OPR}^{\mathrm{H}_{2}}\right)$ are more than $10 t_{\mathrm{ff}}$ (recall $t_{\mathrm{ff}}$ is the local free-fall time at a given density). Thus highly deuterated cores, i.e. with $D_{\text {frac }}^{\mathrm{N}_{2} \mathrm{H}^{+}} \gtrsim 0.1$, that have such densities and that also satisfy the other fiducial parameters and assumed initial conditions would, in the context of the assumption of constant (or slow) density evolution, need to be "dynamically old", i.e. have existed at the current density for significantly longer than their local free-fall time. Below, in \$3.6, we will also place constraints for such cores in the context of dynamicallyevolving densities.

Panel (m) (and panels n, o, p) also show the ambipolar diffusion timescale, $t_{\mathrm{ad}}$, which is expected to be the relevant collapse timescale in magnetically subcritical cores. It is always longer than $t_{\mathrm{ff}}$. The ambipolar diffusion timescale is discussed in more detail in $\$ 4.2$.

\subsubsection{Dependence on $T$}

As shown in panel (j) of Figure 6, between 5 and $15 \mathrm{~K}$, the $D_{\text {frac,eq }}^{\mathrm{N}_{2} \mathrm{H}^{+}}$profile is quite flat. Above $15 \mathrm{~K}, D_{\text {frac,eq }}^{\mathrm{N}_{2} \mathrm{H}^{+}}$ drops down by almost 2 orders of magnitude as $T$ approaches $30 \mathrm{~K}$. The maximum $D_{\text {frac,eq }}^{\mathrm{N}_{2} \mathrm{H}^{+}}$of 0.19 is achieved at $T=13 \mathrm{~K}$. The profile of $t_{\mathrm{eq}, 90}\left(D_{\mathrm{frac}}^{\mathrm{N}_{2} \mathrm{H}^{+}}\right)$is also quite flat across the explored temperatures (panel $n$ ). We find $t_{\text {eq,90 }}\left(D_{\text {frac }}^{\mathrm{N}_{2} \mathrm{H}^{+}}\right)$is always greater than $10 t_{\mathrm{ff}}$ except for the highest temperatures (as is $t_{\mathrm{eq}, 90}\left(\mathrm{OPR}^{\mathrm{H}_{2}}\right)$ ). At $T \lesssim 15 \mathrm{~K}$, $\mathrm{OPR}_{\mathrm{eq}}^{\mathrm{H}_{2}}$ is well below 0.001 , but it goes up quickly at higher temperatures. Regions warmer than $20 \mathrm{~K}$, as, for example, gas in the proximity of young stellar objects, will then experience an increase of the ortho-to-para $\mathrm{H}_{2}$ ratio and thus a drop in the deuterium fraction (in agreement with findings by Fontani et al. 2011 in high-mass star-forming regions and Emprechtinger et al. 2009 in low-mass star-forming regions).

As stated previously, $D_{\text {frac,eq }}^{\mathrm{N}_{2} \mathrm{H}^{+}}$(as well as the deuterium fraction of other deuterated species) is controlled by $\mathrm{OPR}_{\text {eq }}^{\mathrm{H}_{2}}$. As $T$ goes down to $13 \mathrm{~K}, \mathrm{OPR}_{\text {eq }}^{\mathrm{H}_{2}}$ drops (panel (b) of Figure 66, and $D_{\text {frac,eq }}^{\mathrm{N}_{2} \mathrm{H}^{+}}$(panel (j) of Figure 60 increases. Below $13 \mathrm{~K}$, both $\mathrm{OPR}_{\text {eq }}^{\mathrm{H}_{2}}$ and $D_{\text {frac,eq }}^{\mathrm{N}_{2} \mathrm{H}^{+}}$become roughly constant. This is because the $\mathrm{H}_{3}^{+}+\mathrm{H}_{2}$ reacting system contains reactions with activation energies that convert $\mathrm{p}-\mathrm{H}_{2}$ to $\mathrm{o}-\mathrm{H}_{2}$, such as:

$$
\begin{aligned}
& \mathrm{o}-\mathrm{H}_{3}^{+}+\mathrm{p}-\mathrm{H}_{2} \rightarrow \mathrm{p}-\mathrm{H}_{3}^{+}+\mathrm{o}-\mathrm{H}_{2} \\
& \mathrm{o}-\mathrm{H}_{3}^{+}+\mathrm{p}-\mathrm{H}_{2} \rightarrow \mathrm{o}-\mathrm{H}_{3}^{+}+\mathrm{o}-\mathrm{H}_{2} .
\end{aligned}
$$

The activation energy barrier of the above reactions are of the order of $100 \mathrm{~K}$. As the temperature drops, the endothermic channels are effectively closed off and the abundances of both $\mathrm{p}-\mathrm{H}_{2}$ and $\mathrm{o}-\mathrm{H}_{2}$ are controlled mainly by $T$-independent reactions, e.g.,

$$
\mathrm{p}-\mathrm{H}_{2}^{+}+\mathrm{p}-\mathrm{H}_{2} \rightarrow \mathrm{p}-\mathrm{H}_{3}^{+}+\mathrm{H}
$$

Consequently, the $\mathrm{OPR}_{\mathrm{eq}}^{\mathrm{H}_{2}}$ and $D_{\text {frac,eq }}^{\mathrm{N}_{2} \mathrm{H}^{+}}$become almost independent of $T$.

\subsubsection{Dependence on $\zeta$}

$D_{\text {frac,eq }}^{\mathrm{N}_{2} \mathrm{H}^{+}}$drops by a factor of 6 as $\zeta$ increases from $10^{-18}$ to $10^{-15} \mathrm{~s}^{-1}$ (panel $\mathrm{k}$ ), due to the enhanced electron abundance and the consequent dissociative recombination of the deuterated isotopologues of $\mathrm{H}_{3}^{+}$(see also 


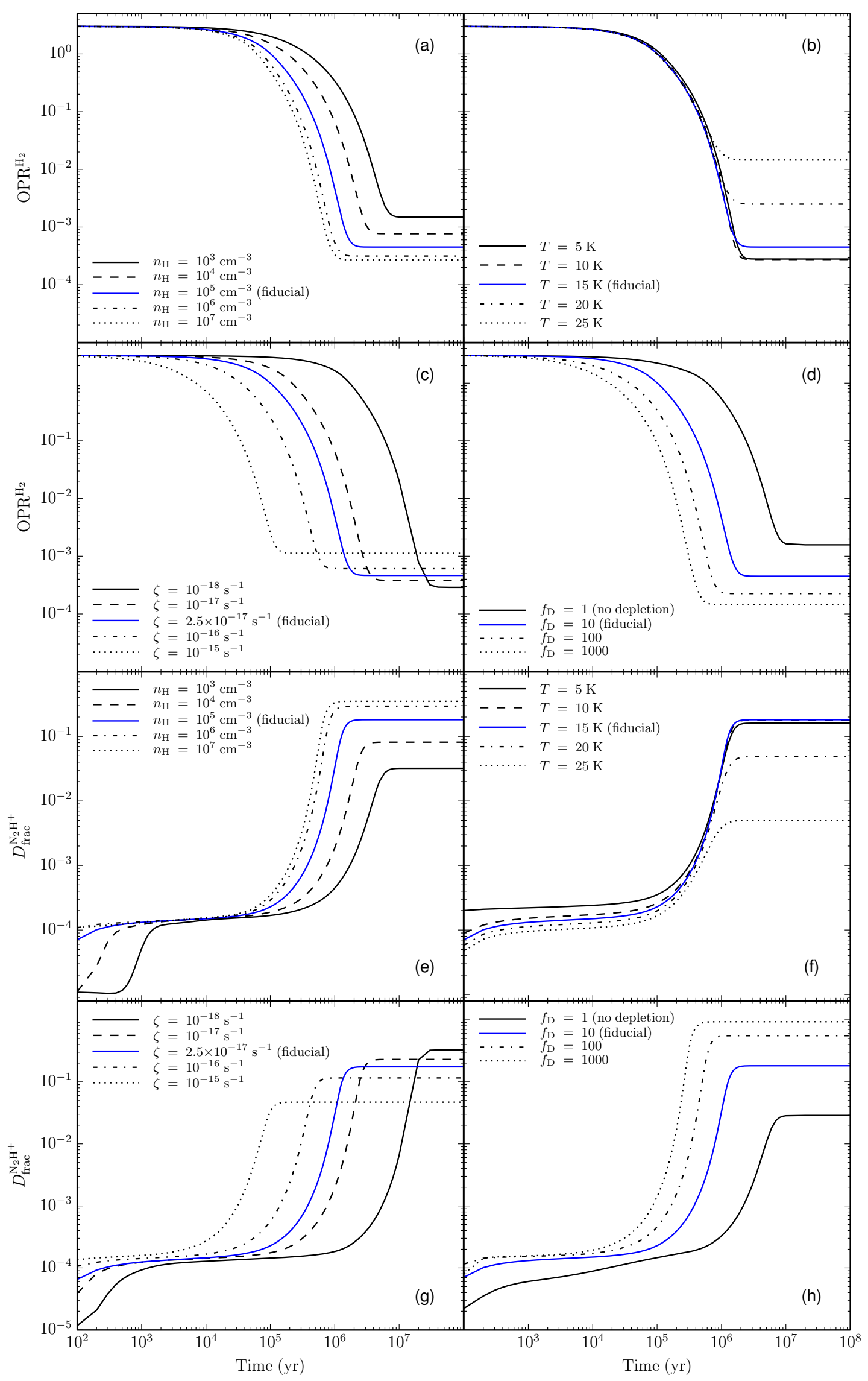

Figure 5. Time evolution of $\mathrm{OPR}^{\mathrm{H}_{2}}$ (panels a to d) and $D_{\text {frac }}^{\mathrm{N}_{2} \mathrm{H}^{+}}$(panels e to h) for various densities (a, e), temperatures (b, f), cosmic ray ionization rates $(\mathrm{c}, \mathrm{g})$ and depletion factors $(\mathrm{d}, \mathrm{h})$. The blue solid lines correspond to the fiducial model (as in Fig. 2). In each case of exploring the effect of varying a particular parameter, the other parameter values are set to those of the fiducial model. See $\$ 3.4$ for the complete description of the exploration. 


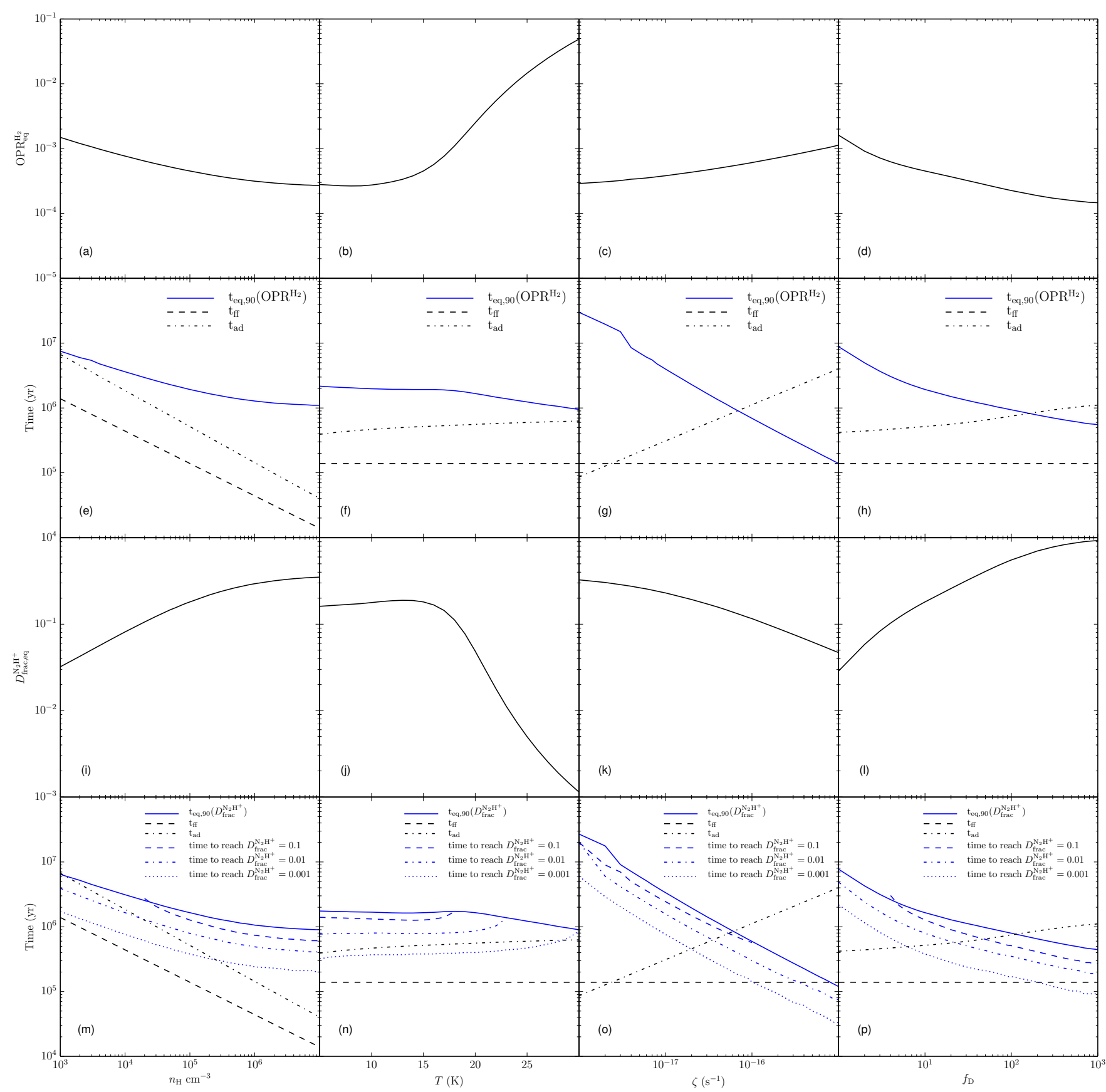

Figure 6. Parameter-space exploration of dependence of $\mathrm{OPR}_{\mathrm{eq}}^{\mathrm{H}_{2}}$ (top row), $t_{\mathrm{eq}, 90}\left(\mathrm{OPR}^{\mathrm{H}_{2}}\right)(2 \mathrm{nd}$ row $), D_{\text {frac,eq }}^{\mathrm{N}_{2} \mathrm{H}^{+}}(3 \mathrm{rd}$ row $), t_{\mathrm{eq}, 90}\left(D_{\text {frac }}^{\mathrm{N}_{2} \mathrm{H}^{+}}\right)$ (bottom row) as a function of density $n_{\mathrm{H}}$ (left column), temperature $T$ (2nd column), cosmic ray ionization rate $\zeta$ (3rd column), and depletion factor $f_{\mathrm{D}}$ (right column) (see $\$ 3.2$ for definitions). In the 4 th row, we also show the times to reach $D_{\text {frac }}^{\mathrm{N}_{2} \mathrm{H}^{+}}=0.1,0.01,0.001$ (missing portions of the lines imply $D_{\text {frac }}^{\mathrm{N}_{2} \mathrm{H}^{+}}$does not reach the value of interest for these conditions). Also shown are free-fall time $t_{\mathrm{ff}}$ (Eq. 8 and ambipolar diffusion time $t_{\mathrm{ad}}\left(\$ 4.2\right.$ to be compared to $t_{\mathrm{eq}, 90}\left(\mathrm{OPR}^{\mathrm{H}_{2}}\right)$ and $t_{\mathrm{eq}, 90}\left(D_{\mathrm{frac}}^{\mathrm{N}_{2} \mathrm{H}^{+}}\right)$. 


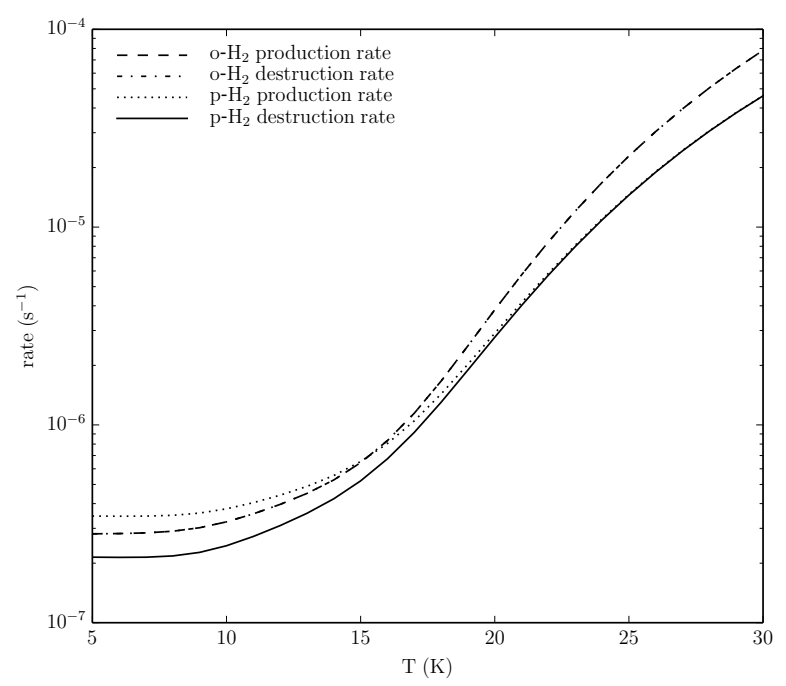

Figure 7. Production and destruction rates of o- $\mathrm{H}_{2}$ and $\mathrm{p}-\mathrm{H}_{2}$ at equilibrium time step as a function of T. See 33.4 .2

Caselli et al. 2008). The highest $D_{\text {frac,eq }}^{\mathrm{N}_{2} \mathrm{H}^{+}}$of 0.33 appears at the lowest $\zeta=10^{-18} \mathrm{~s}^{-1}$. This shows the importance for the astrochemical modeling of constraining $\zeta$. Panel (o) shows that $t_{\mathrm{eq}, 90}\left(D_{\mathrm{frac}}^{\mathrm{N}_{2} \mathrm{H}^{+}}\right)$changes by more than 2 orders of magnitude within the $\zeta$ range explored. The smallest $t_{\text {eq }, 90}\left(D_{\text {frac }}^{\mathrm{N}_{2} \mathrm{H}^{+}}\right)$is only $1.2 \times 10^{5}$ yr at $\zeta=$ $10^{-15} \mathrm{~s}^{-1}$, which is much shorter than $t_{\mathrm{ff}}$. However, such high CR ionization rates are not expected to be relevant in typical Galactic star-forming regions (and would also be expected to yield relatively high kinetic temperatures $)$. With moderate $\zeta\left(\lesssim 10^{-16} \mathrm{~s}^{-1}\right), t_{\mathrm{eq}, 90}\left(D_{\mathrm{frac}}^{\mathrm{N}_{2} \mathrm{H}^{+}}\right)$ is significantly greater than $t_{\mathrm{ff}}\left(\gtrsim 7 t_{\mathrm{ff}}\right)$ at the fiducial density. The dependence of $\mathrm{OPR}^{\mathrm{H}_{2}}$ with $\zeta$ is shown in panel (c) and its equilibrium timescale in panel (g).

\subsubsection{Dependence on $f_{\mathrm{D}}$}

Panel (l) of Figure 6 shows that $D_{\text {frac,eq }}^{\mathrm{N}_{2} \mathrm{H}^{+}}$goes up by more than an order of magnitude as $f_{\mathrm{D}}$ increases from 1 to 1000 . This agrees with the expectation that depletion of neutral species, in particular $\mathrm{CO}$ and $\mathrm{O}$, the main destruction partners of $\mathrm{H}_{3}^{+}$and its deuterated forms, will result in the enhancement of $D_{\text {frac }}^{\mathrm{N}_{2} \mathrm{H}^{+}}$(see also Dalgarno \& Lepp 1984). At $f_{\mathrm{D}}=1000$ we encounter the highest $D_{\text {frac,eq }}^{\mathrm{N}_{2} \mathrm{H}^{+}}=0.93$ in our exploration. Such high values have seldom been observed (e.g., Miettinen et al.|2012). We find $t_{\text {eq,90 }}\left(D_{\text {frac }}^{\mathrm{N}_{2} \mathrm{H}^{+}}\right)$decreases with stronger depletion, which is shown in panel $(\mathrm{p})$, although $t_{\mathrm{eq}, 90}\left(D_{\mathrm{frac}}^{\mathrm{N}_{2} \mathrm{H}^{+}}\right)$is at least a factor 7 larger than $t_{\mathrm{ff}}$ when $f_{\mathrm{D}} \lesssim 100$ at the fiducial density.

An interesting point is whether $D_{\text {frac,eq }}^{\mathrm{N}_{2} \mathrm{H}^{+}}$will keep going up with stronger depletion. We extend our exploration to $f_{\mathrm{D}}=10^{6}$, which is shown in Fig. 8. We can see in panel (b), the $D_{\text {frac,eq }}^{\mathrm{N}_{2} \mathrm{H}^{+}}-f_{\mathrm{D}}$ relation drops moderately at $f_{\mathrm{D}} \sim 2000$. In panel $(\mathrm{a}),\left[\mathrm{H}_{3}^{+}\right]$and $\left[\mathrm{H}_{2} \mathrm{D}^{+}\right]$reach the peak at $f_{\mathrm{D}} \sim 2000$ and drop moderately until $f_{\mathrm{D}}=10^{6}$. Besides the destruction partners like CO, electrons can also destroy $\mathrm{H}_{3}^{+}$and its deuterated forms. We plot the electron abundance versus $f_{\mathrm{D}}$ in Fig. 8(a). As we can see, the electron abundance increases at $f_{\mathrm{D}} \gtrsim 2000$ while $D_{\text {frac,eq }}^{\mathrm{N}_{2} \mathrm{H}^{+}}$drops. This supports our expectation that the super-depletion of heavy elements reduces the destruction partners of electron, so that [e-] can approach a high level which suppresses the abundances of $\mathrm{H}_{3}^{+}$and $\mathrm{H}_{2} \mathrm{D}^{+}$, etc. To confirm this, we remove all dissociative recombination reactions between electron and O-bearing species $(\sim 40$ reactions. These species contain no Nitrogen or Carbon $\sqrt{3}$ and perform the exploration again. The results are shown in Fig. 9. Now we see that the bump of $D_{\text {frac,eq }}^{\mathrm{N}_{2} \mathrm{H}^{+}}$around $f_{\mathrm{D}} \sim 2000$ is gone. $D_{\text {frac,eq }}^{\mathrm{N}_{2} \mathrm{H}^{+}}$simply increases with $f_{\mathrm{D}}$ and reaches a constant value $(\sim 0.7)$. In panel (a) of Fig. 9, because of the reduced number of dissociative reactions, the electron abundance is high at moderate depletion, as compared to Fig. 8(a). The [e-] and $D_{\text {frac,eq }}^{\mathrm{N}_{2} \mathrm{H}^{+}}$at extreme $f_{\mathrm{D}}$ approach the same values as those in Fig. 8, respectively. These imply that what we see in panel (b) of Fig. 8 is the result of the competition between two mechanisms: (1) species like CO can destroy $\mathrm{H}_{3}^{+}$and its deuterated forms; (2) O-bearing species consume electron through dissociative reactions. It turns out that in our exploration of $f_{\mathrm{D}}$ (panel $\mathrm{b}$ of Fig. 8D, mechanism (1) dominates at $f_{\mathrm{D}} \lesssim 2000$, and mechanism $(2)$ dominates at $2000 \lesssim f_{\mathrm{D}} \lesssim 10000$. At $f_{\mathrm{D}}$ $\gtrsim 10000$, there are too few O-bearing species consuming electron, explaining the $D_{\text {frac,eq }}^{\mathrm{N}_{2} \mathrm{H}^{+}}-f_{\mathrm{D}}$ relation in Fig. $9(\mathrm{~b})$.

\subsubsection{Dependence on Initial $O P R^{\mathrm{H}_{2}}$}

The effect of varying the initial $\mathrm{OPR}^{\mathrm{H}_{2}}$ on the time evolution of the fiducial model was discussed above in \$3.3.2. In Figure 10 we show the effect on the deuteration timescale parameter space $\left(n_{\mathrm{H}}, T, \zeta, f_{\mathrm{D}}\right)$ exploration of starting with $\mathrm{OPR}^{\mathrm{H}_{2}}=1,0.1,0.01$, rather than 3 .

In general, the effect of a lower starting $\mathrm{OPR}^{\mathrm{H}_{2}}$ value is to reduce the timescales needed to reach a given level of $D_{\text {frac }}^{\mathrm{N}_{2} \mathrm{H}^{+}}$. However, for most of the parameter space, $t_{\text {eq,90 }}\left(D_{\mathrm{frac}}^{\mathrm{N}_{2} \mathrm{H}^{+}}\right)$still remains significantly greater than $t_{\mathrm{ff}}$.

\subsubsection{Highest $D_{\text {frac }}^{\mathrm{N}_{2} \mathrm{H}^{+}}$predicted in our model}

High values of $D_{\text {frac }}^{\mathrm{N}_{2} \mathrm{H}^{+}}$have been reported in recent observations of starless cores. Fontani et al. (2011) observed several potential massive starless cores, finding a highest $D_{\text {frac }}^{\mathrm{N}_{2} \mathrm{H}^{+}}=0.7$ in their source Infrared Dark Cloud G2. An even higher value of $D_{\text {frac }}^{\mathrm{N}_{2} \mathrm{H}^{+}}=0.99$ has been reported by Miettinen et al. (2012, though this may be affected by the uncertainties from treating $\mathrm{N}_{2} \mathrm{H}^{+}$with non-LTE model but $\mathrm{N}_{2} \mathrm{D}^{+}$with LTE model), toward Orion B9 SMM1. Such high values are not predicted by our fiducial model.

\footnotetext{
3 The reason we choose O-bearing species is that we have made another three explorations where we reduce initial $[\mathrm{C}],[\mathrm{N}],[\mathrm{O}]$ independently. We denote the depletion of $\mathrm{C}, \mathrm{N}, \mathrm{O}$ with $f_{\mathrm{D}}(\mathrm{C})$, $f_{\mathrm{D}}(\mathrm{N}), f_{\mathrm{D}}(\mathrm{O})$, respectively. We find from the explorations that reducing initial $[\mathrm{O}]$ can reproduce the drop of $D_{\text {frac,eq }}^{\mathrm{N}_{2} \mathrm{H}^{+}}$. So we expect that O-bearing species play the crucial role.
} 


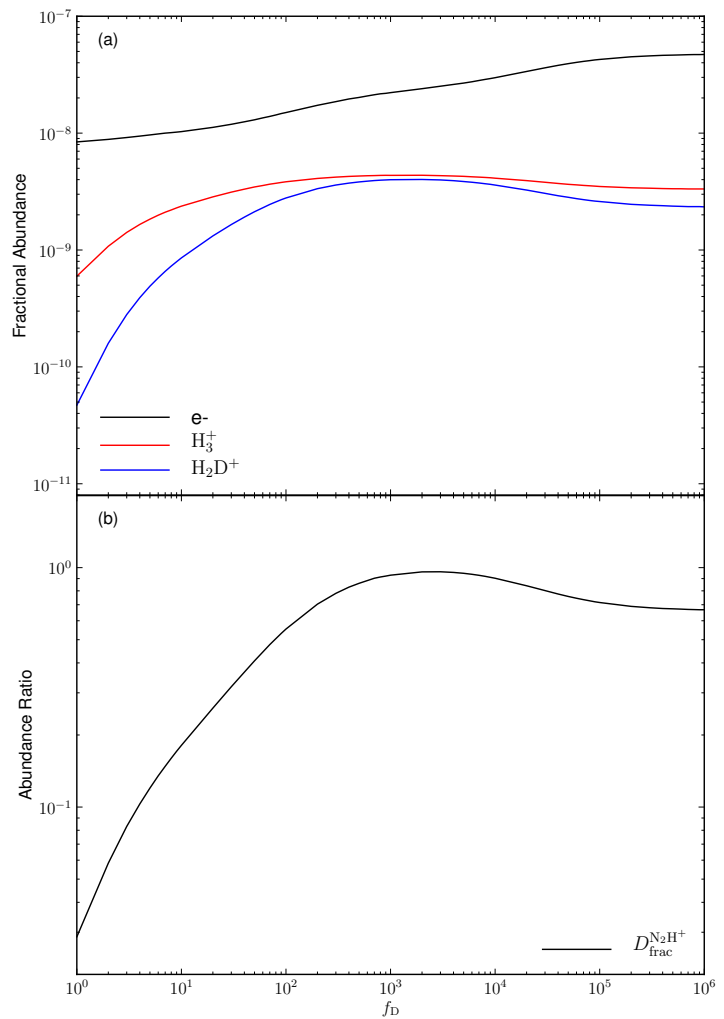

Figure 8. (a) Relations between the depletion factor and the fractional abundances (relative to $n_{\mathrm{H}}$ ) of $\mathrm{H}_{3}^{+}, \mathrm{H}_{2} \mathrm{D}^{+}$, and electron, respectively. The abundances are taken at the equilibrium step of $D_{\text {frac,eq }}^{\mathrm{N}_{2} \mathrm{H}^{+}}$. (b) Same as the panel (l) in Fig. 6 but extended to $f_{\mathrm{D}}$ $=10^{6}$.

However, it is interesting if we combine the explored parameters $n_{\mathrm{H}}, T, \zeta, f_{\mathrm{D}}$ at the values where $D_{\text {frac,eq }}^{\mathrm{N}_{2} \mathrm{H}^{+}}$peaks $\left(n_{\mathrm{H}}=10^{7} \mathrm{~cm}^{-3}, T=13 \mathrm{~K}, \zeta=10^{-18} \mathrm{~s}^{-1}, f_{\mathrm{D}}=1000\right)$ to gauge the maximum level of deuteration that can result from our model (might not be global in core). Equilibrium ratios and timescales are summarized in Table 3. We find $D_{\text {frac }}^{\mathrm{N}_{2} \mathrm{H}^{+}}$goes up to 0.903 , while $t_{\text {eq, } 90}\left(D_{\text {frac }}^{\mathrm{N}_{2} \mathrm{H}^{+}}\right)$is about $46 t_{\mathrm{ff}}\left(1.39 \times 10^{4} \mathrm{yr}\right.$ at $\left.n_{\mathrm{H}}=10^{7} \mathrm{~cm}^{-3}\right)$. Detailed constraints on the parameter space needed for individual observed sources will be presented in a future study.

\subsection{Effect of Time-Dependent Depletion/Desorption}

In Figure 11 we compare the fiducial model (with constant $f_{\mathrm{D}}=10$ ) and the TDD model (with starting values of $f_{\mathrm{D}}=1$ and $f_{\mathrm{D}}=10$ ). Panel (a) shows the time evolution of the fractional abundances of $\mathrm{N}_{2} \mathrm{D}^{+}, \mathrm{N}_{2} \mathrm{H}^{+}, \mathrm{CO}$, and $\mathrm{N}_{2}$. The time evolution of the abundance of these species show qualitatively similar behaviours in the two models, with modest quantitative differences. We note that the TDD models do not reach equilibrium within $10^{8} \mathrm{yr}$ because of continuing freeze-out, especially of $\mathrm{N}_{2}$. The $\mathrm{N}_{2} \mathrm{D}^{+}$abundance shows a plateau between $5 \times 10^{6}$ and $5 \times 10^{7}$ yr, before dropping together with the $\mathrm{N}_{2}$ abundance.

In panel (b) we compare gas-phase $\mathrm{OPR}^{\mathrm{H}_{2}}$ and $D_{\text {frac }}^{\mathrm{N}_{2} \mathrm{H}^{+}}$ between the fiducial model and the TDD models. Compared to the fiducial model, the decline of $\mathrm{OPR}^{\mathrm{H}_{2}}$ is slower in the $f_{\mathrm{D}}=1$ TDD model and faster in the $f_{\mathrm{D}}=10$ TDD model, so that for most of the time evolution, up

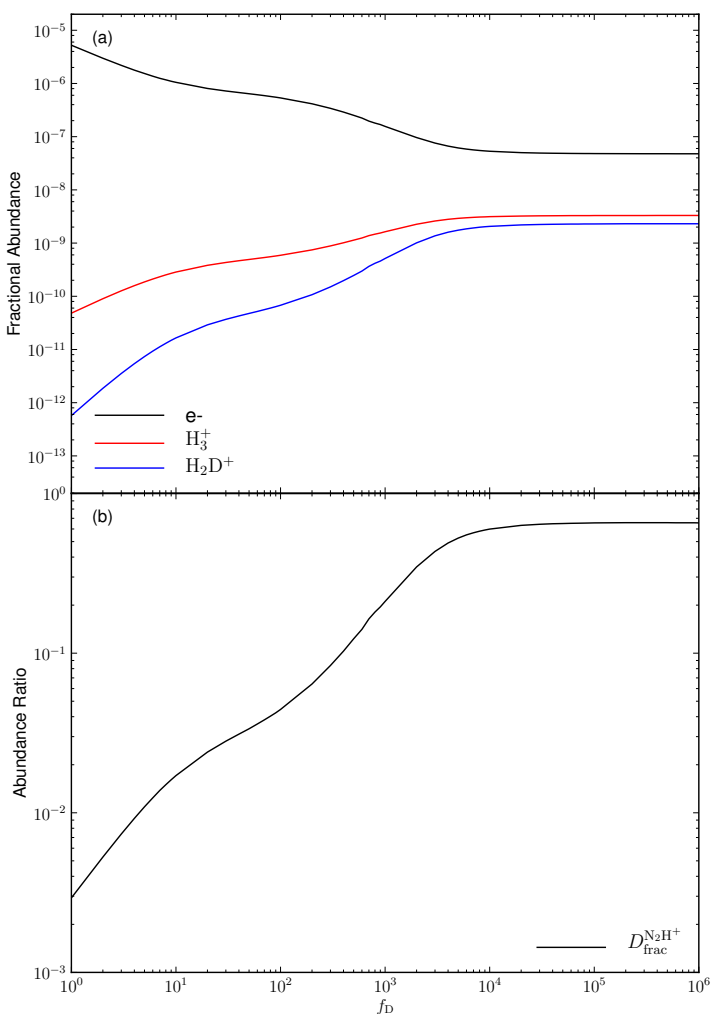

Figure 9. Same as Fig. 8 but now all dissociative recombination reactions between electron and O-bearing species (containing no Nitrogen or Carbon) are removed ( $\sim 40$ reactions). This is used to prove that the "bump" in panel (b) of Fig. 8 is caused by these reactions (no "bump" after removing the reactions). See 3.4 .4

to $\sim 10^{7} \mathrm{yr}$, the fiducial models results are bracketed by the TDD models.

Note our simple TDD models do not include surface chemistry, since this opens up even larger uncertainties, which we defer to a future study. As an initial check to see if surface chemistry can have a significant effect, we have examined the S13 models with and without surface reactions. The effect of including surface chemistry within these models on $\mathrm{OPR}^{\mathrm{H}_{2}}$ is very minor, and the time evolution of $D_{\text {frac }}^{\mathrm{N}_{2} \mathrm{H}^{+}}$is also largely unaffected. Thus we do not expect our fiducial or TDD model results to be significantly affected by neglect of surface chemistry.

\subsection{Effect of Dynamical Density Evolution}

We have so far presented models that treat density as an unchanging, controllable parameter. Here we carry out a set of Dynamical Density Evolution (DDE) models that examine various rates of collapse relative to the freefall rate by which a core of current density $n_{\mathrm{H}, 1}$ at current time $t_{1}$ is created from a core at starting density $n_{\mathrm{H}, 0}$ at starting time $t_{0}$. We parameterize the rate of density increase via

$$
\frac{\mathrm{d} n_{\mathrm{H}}}{\mathrm{d} t}=\alpha_{\mathrm{ff}} \frac{n_{\mathrm{H}}(t)}{t_{\mathrm{ff}}(t)}
$$

where $t_{\mathrm{ff}}$ is the local free-fall time at current density $n_{\mathrm{H}}$ (Eq. 8) and $\alpha_{\mathrm{ff}}$ is a parameter controlling how fast the core collapses. We define a past time variable that increases going back into a core's history via

$$
t_{\text {past }}=t_{1}-t \text {. }
$$




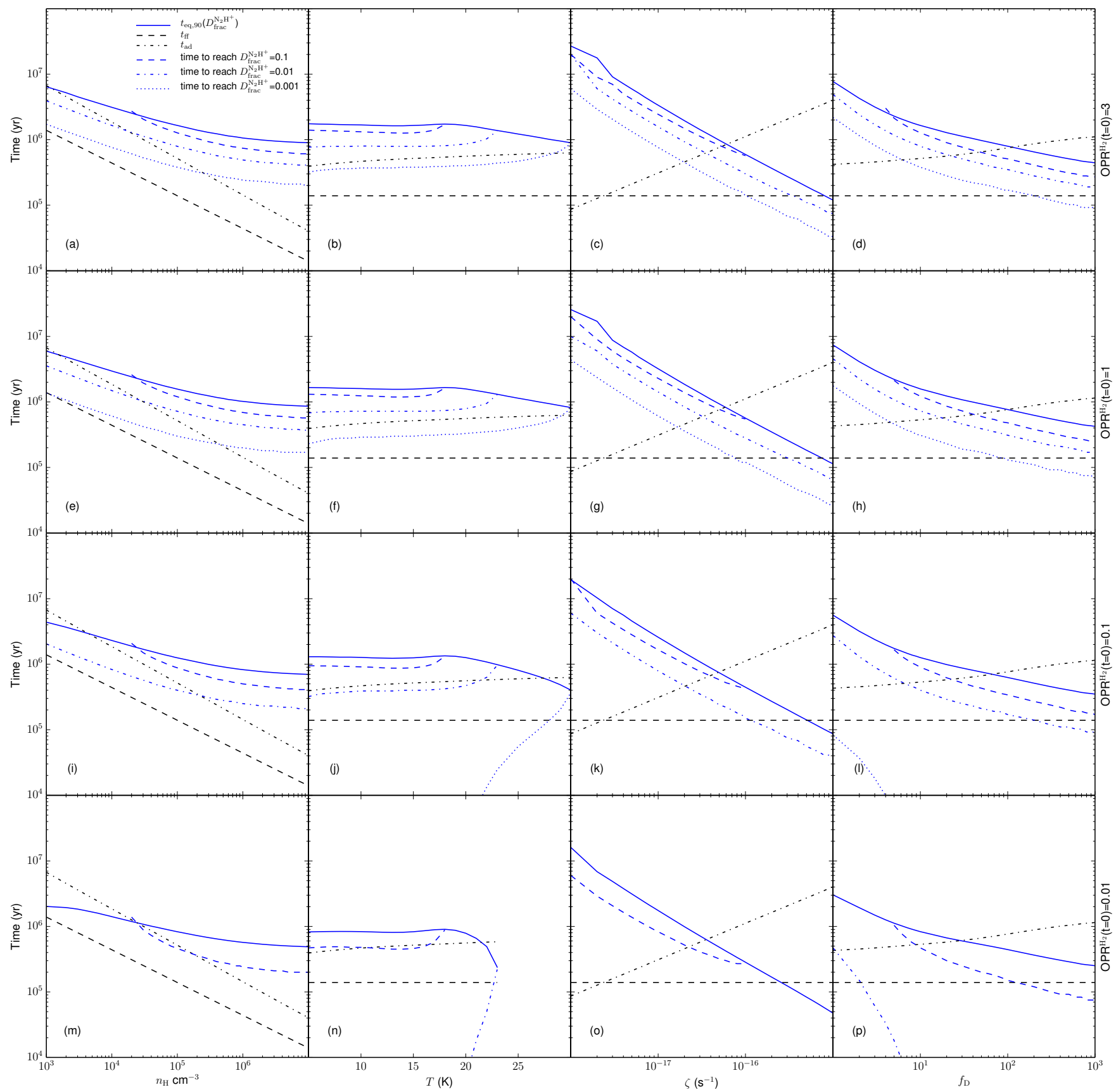

Figure 10. Same as bottom row of Fig. 6 which now appears as the top row here. Then the 2nd, 3rd and bottom rows show the effect of changing the initial $\mathrm{OPR}^{\mathrm{H}_{2}}$ to $1,0.1$ and 0.01 , respectively. The blank parts in high-temperature exploration of the bottom row are due to this initial $\mathrm{OPR}^{\mathrm{H}_{2}}=0.01$ being smaller than $\mathrm{OPR}_{\text {eq }}^{\mathrm{H}_{2}}$ (see panel (b) in Fig. 6).

So the past density evolution is described by

$n_{\mathrm{H}, \text { past }}=n_{\mathrm{H}, 1}\left[1+3.60 \alpha_{\mathrm{ff}}\left(\frac{n_{\mathrm{H}, 1}}{10^{5} \mathrm{~cm}^{-3}}\right)^{1 / 2}\left(\frac{t_{\text {past }}}{10^{6} \mathrm{yr}}\right)\right]^{-2}$.

For a given current "target" density, $n_{\mathrm{H}, 1}$, we then explore different ratios of starting density: $n_{\mathrm{H}, 0} / n_{\mathrm{H}, 1}=$ $0.1,0.01$ and three different values of $\alpha_{\mathrm{ff}}=0.01,0.1,1$. We run these models for three different target densities $n_{\mathrm{H}, 1}=10^{4}, 10^{5}, 10^{6} \mathrm{~cm}^{-3}$. We first start by keeping other aspects of the modeling the same as the fiducial model, i.e. a starting $\mathrm{OPR}_{\mathrm{eq}}^{\mathrm{H}_{2}}=3$ and a fixed depletion factor of $f_{\mathrm{D}}=10$. The results are shown in Figure 12 .

The first row of Figure 12 shows the density evolution with $t_{\text {past }}$ increasing to the left. For each $n_{\mathrm{H}, 1}$, the faster the collapse rate (larger $\left.\alpha_{\mathrm{ff}}\right)$, the shorter the past history of the core since its starting condition. Similarly, for fixed $n_{\mathrm{H}, 1}$ and $\alpha_{\mathrm{ff}}$, larger values of $n_{\mathrm{H}, 0}$ mean shorter core histories. The second row of Figure 12 shows the evolution of the ionization fraction, which declines as density increases. The third row shows the evolution of $\mathrm{OPR}_{\mathrm{eq}}^{\mathrm{H}_{2}}$, showing rapid falls from the assumed starting value of 


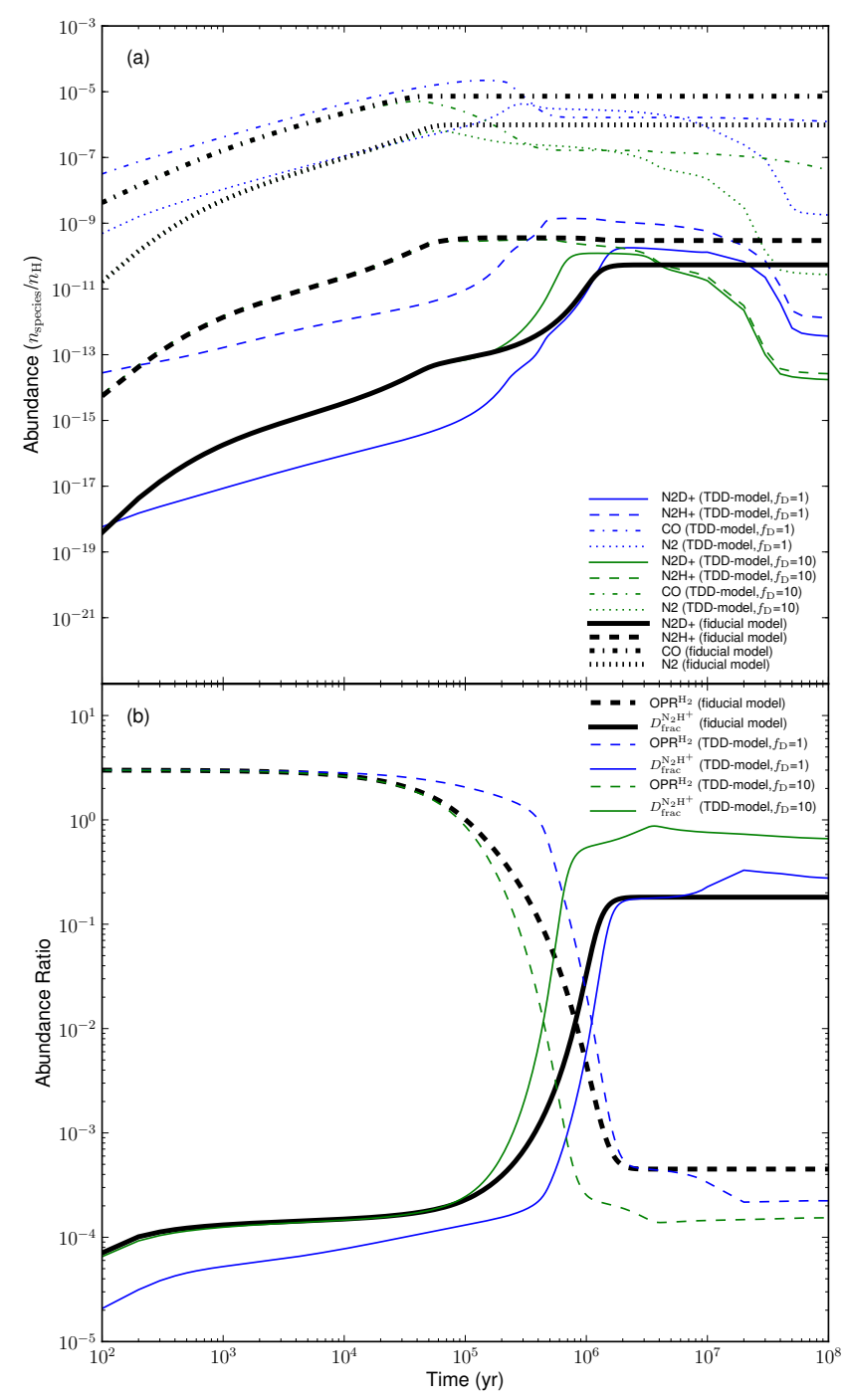

Figure 11. (a) Top panel: Time evolution of fractional abundances of important gas-phase species in both the fiducial model (thick black lines) and the TDD models (thin lines). The two TDD models shown here started with $f_{\mathrm{D}}=1$ (blue) and $f_{\mathrm{D}}=10$ (green). (b) Bottom panel: Time evolution of gas-phase $\mathrm{OPR}^{\mathrm{H}_{2}}$ and $D_{\text {frac }}^{\mathrm{N}_{2} \mathrm{H}^{+}}$in the fiducial model (thick black lines) and the TDD model (thin lines). The two TDD models shown here started with $f_{\mathrm{D}}=1$ (blue) and $f_{\mathrm{D}}=10$ (green).

3. Note that in some of the fast-evolving, higher density models there is insufficient time for $\mathrm{OPR}_{\mathrm{eq}}^{\mathrm{H}_{2}}$ to reach its equilibrium value. The fourth and fifth rows show the abundances of $\mathrm{N}_{2} \mathrm{H}^{+}$and $\mathrm{N}_{2} \mathrm{D}^{+}$, respectively, while the bottom row shows the evolution of $D_{\text {frac }}^{\mathrm{N}_{2} \mathrm{H}^{+}}$. Again, note that in the fast-evolving, higher density models there is insufficient time to reach $D_{\text {frac,eq }}^{\mathrm{N}_{2} \mathrm{H}^{+}}$.

Note that very slowly evolving models with $t_{\text {past }}$ extending beyond several $\times 10^{7}$ yr are unlikely to be relevant given estimated GMC lifetimes (e.g., $\sim 3 \times 10^{7} \mathrm{yr}$, Williams \& McKee 1997). Considering the cases with the fastest collapse with $\alpha_{\mathrm{ff}}=1$ that create cores with $n_{\mathrm{H}}=10^{5}$ to $10^{6} \mathrm{~cm}^{-3}$ from starting conditions a factor of 10 lower in density (green solid lines in panels (i), (o), (l), (r) of Fig. 12), then the collapse history did not produce very low $\mathrm{OPR}^{\mathrm{H}_{2}}$ or very high $D_{\text {frac }}^{\mathrm{N}_{2} \mathrm{H}^{+}}$(always $<10^{-3}$ ). However, models of fast collapse could potentially form highly deuterated cores if starting from lower densities (thus giving more time for chemical evolution) or, as explored below, with lower initial $\mathrm{OPR}_{\mathrm{eq}}^{\mathrm{H}_{2}}$ ratios.

We next re-run the above DDE models, but with timedependent depletion/desorption starting from $f_{\mathrm{D}}=1$. These TDD + DDE models are shown in Figure 13. We find broadly similar results that rapidly collapsing high density cores have difficulty achieving high levels of $D_{\text {frac }}^{\mathrm{N}_{2} \mathrm{H}^{+}}$.

We next explore the effect of the assumed starting $\mathrm{OPR}_{\text {eq }}^{\mathrm{H}_{2}}$ and the starting depletion factor. Focussing on models with $n_{\mathrm{H}, 1}=10^{5}$ and $10^{6} \mathrm{~cm}^{-3}$ and with $\alpha_{\mathrm{ff}}=0.01,0.033,0.1,0.33,1$, we run TDD $+\mathrm{DDE}$ models for initial $\mathrm{OPR}_{\mathrm{eq}}^{\mathrm{H}_{2}}=0.01,0.1,1,3$ and initial $f_{\mathrm{D}}=1,10,100$, and show their results for $D_{\text {frac }}^{\mathrm{N}_{2} \mathrm{H}^{+}}$in Figures $14,15,16$.

For the models with initial $f_{\mathrm{D}}=1$, we find $D_{\text {frac }}^{\mathrm{N}_{2} \mathrm{H}^{+}}>$ 0.1 cores require $\alpha_{\mathrm{ff}} \lesssim 0.33$, unless the starting $\mathrm{OPR}_{\mathrm{eq}}^{\mathrm{H}_{2}}=0.01$. However, these requirements become more relaxed if we start with $f_{\mathrm{D}}=10,100$. To reconcile models of fast collapse with high deuteration, it would require larger values of initial $f_{\mathrm{D}}(>10)$ and small initial $\mathrm{OPR}_{\text {eq }}^{\mathrm{H}_{2}}$.

In order to see if these models with different starting values of $f_{\mathrm{D}}$ can be separated by absolute abundances of $\left[\mathrm{N}_{2} \mathrm{H}^{+}\right]$, we plot the relation between $D_{\text {frac }}^{\mathrm{N}_{2} \mathrm{H}^{+}}$and $\left[\mathrm{N}_{2} \mathrm{H}^{+}\right]$ with $n_{\mathrm{H}, 1}=10^{5} \mathrm{~cm}^{-3}$ in Figure 17 and $n_{\mathrm{H}, 1}=10^{6} \mathrm{~cm}^{-3}$ in Figure 18. As expected, the absolute abundances are lower in models with higher initial $f_{\mathrm{D}}$, so that observations of these abundances, together with $D_{\text {frac }}^{\mathrm{N}_{2} \mathrm{H}^{+}}$, can help distinguish between the model families.

\section{DISCUSSION}

\subsection{Deuteration as a Chemical Clock}

The high equilibrium values of $D_{\text {frac }}^{\mathrm{N}_{2} \mathrm{H}^{+}}$, together with the small starting fractional abundance of $\mathrm{D}$ relative to $\mathrm{H}$ and the controlling influence of the ortho-to-para ratio of $\mathrm{H}_{2}$, which decays relatively slowly, mean that over a wide range of parameter space relevant for cold, dense starless cores, the timescale to reach deuteration equilibrium is relatively long compared to, for example, the local freefall timescale. Unless the starting conditions for core formation involve extremely low values of $\mathrm{OPR}^{\mathrm{H}_{2}} \lesssim 10^{-2}$, high values of depletion factor $f_{\mathrm{D}} \gtrsim 10$ or suffer high values of cosmic ray ionization $\zeta \gtrsim 10^{-16} \mathrm{~s}^{-1}$, then observing high values of $D_{\text {frac }}^{\mathrm{N}_{2} \mathrm{H}^{+}} \gtrsim 0.1$ implies that the core is contracting at rates significantly lower than free-fall, so that it has been in a dense, cold state for at least several dynamical times. Note that if the core is close to chemical equilibrium, then the derived deuteration timescale is only a lower limit to its age. More accurate constraints require tailored application of chemical models to particular physical conditions of individual cores and may require measurement of absolute abundances to constrain the effects of the depletion factor.

In one of the best studied low-mass pre-stellar cores, L1544 in the Taurus molecular cloud, we can attempt to contrain an age. Within the central $3600 \mathrm{AU}$ (the beam size of the IRAM $30 \mathrm{~m}$ antenna at the frequency of 


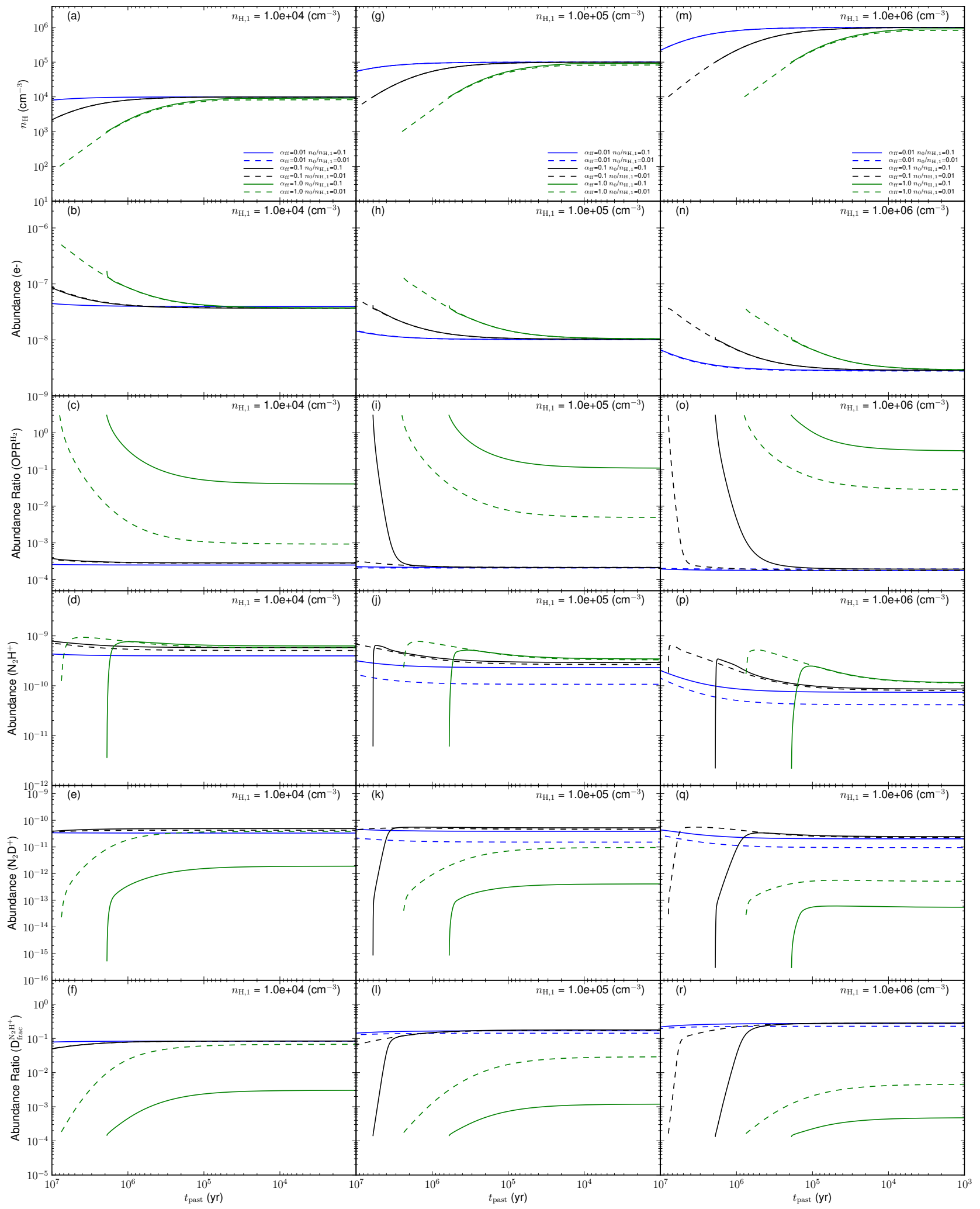

Figure 12. Dynamical Density Evolution (DDE) models that have a time-evolving density at various rates relative to free-fall collapse, as parameterized by $\alpha_{\mathrm{ff}}$ (see Eq. 14). Each column shows the results of particular target densities $n_{\mathrm{H}, 1}=10^{4}, 10^{5}, 10^{6} \mathrm{~cm}{ }^{-3}$ (left to right). The top row shows the time evolution of the density as a function of $t_{\text {past }}$, increasing to the left. In each case, models with $\alpha_{\mathrm{ff}}=0.01,0.1,1$ and starting to final density ratios of $n_{\mathrm{H}, 0} / n_{\mathrm{H}, 1}=0.1,0.01$ are shown. Then, rows 2-6 show the time evolution of [e-], OPR $\mathrm{H}_{2},\left[\mathrm{~N}_{2} \mathrm{H}^{+}\right]$, $\left[\mathrm{N}_{2} \mathrm{D}^{+}\right], D_{\text {frac }}^{\mathrm{N}_{2} \mathrm{H}^{+}}$, respectively. 


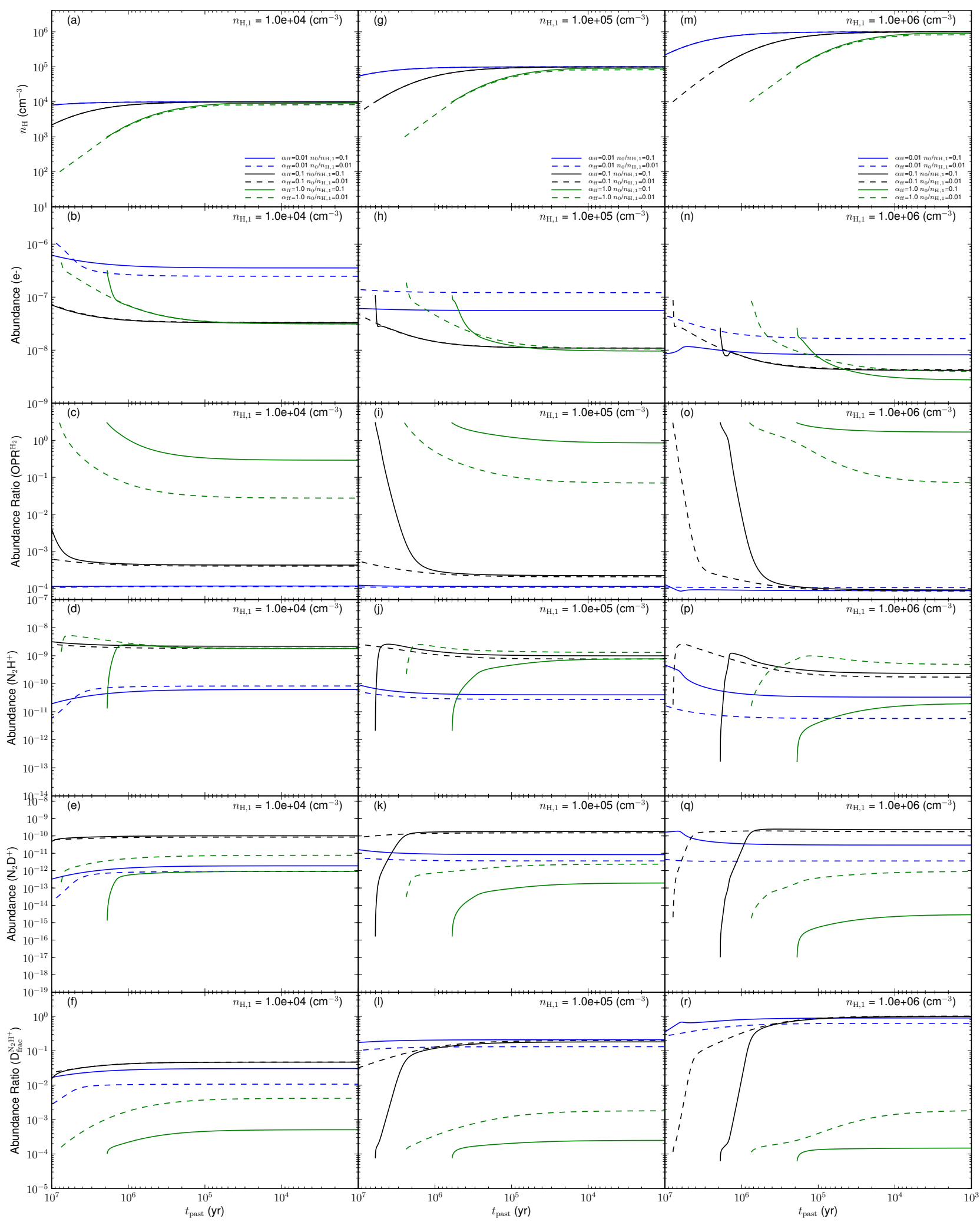

Figure 13. Same as Fig. 12 but all models are now with Time-Dependent Depletion/Desorption (TDD). 


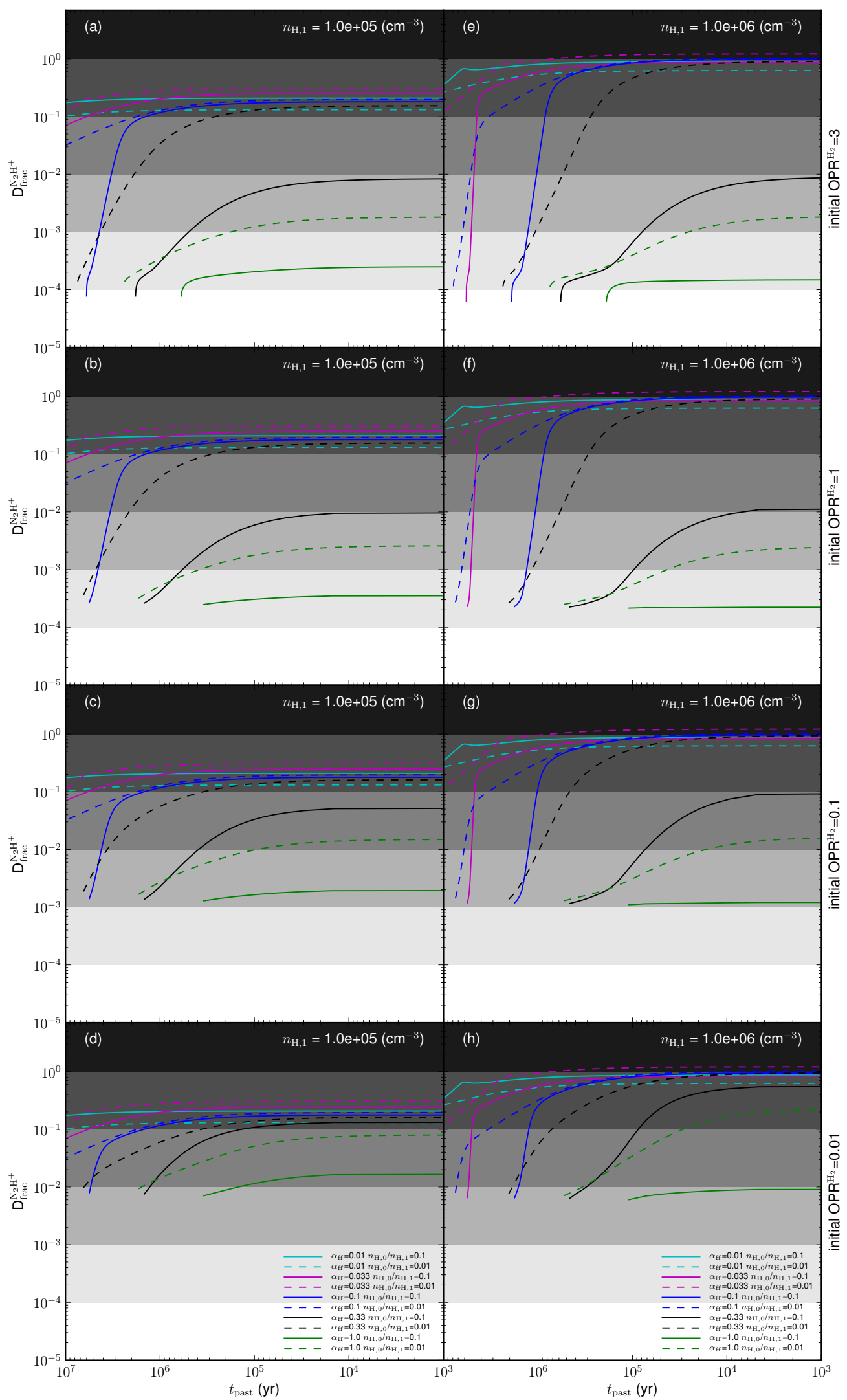

Figure 14. Effect of starting $\mathrm{OPR}^{\mathrm{H}_{2}}$ on $D_{\text {frac }}^{\mathrm{N}_{2} \mathrm{H}^{+}}$in dynamical density evolution with time-dependent depletion/desorption (DDE+TDD) models of dense cores. Left and right columns show the results of target densities $n_{\mathrm{H}, 1}=10^{5}, 10^{6} \mathrm{~cm}^{-3}$, respectively. From top to bottom, the rows show starting $\mathrm{OPR}^{\mathrm{H}_{2}}=3,1,0.1,0.01$, respectively. In each case, models with $\alpha_{\mathrm{ff}}=0.01,0.033,0.1,0.33,1$ and starting to final density ratios of $n_{\mathrm{H}, 0} / n_{\mathrm{H}, 1}=0.1,0.01$ are shown. Here the starting $f_{\mathrm{D}}=1$. 


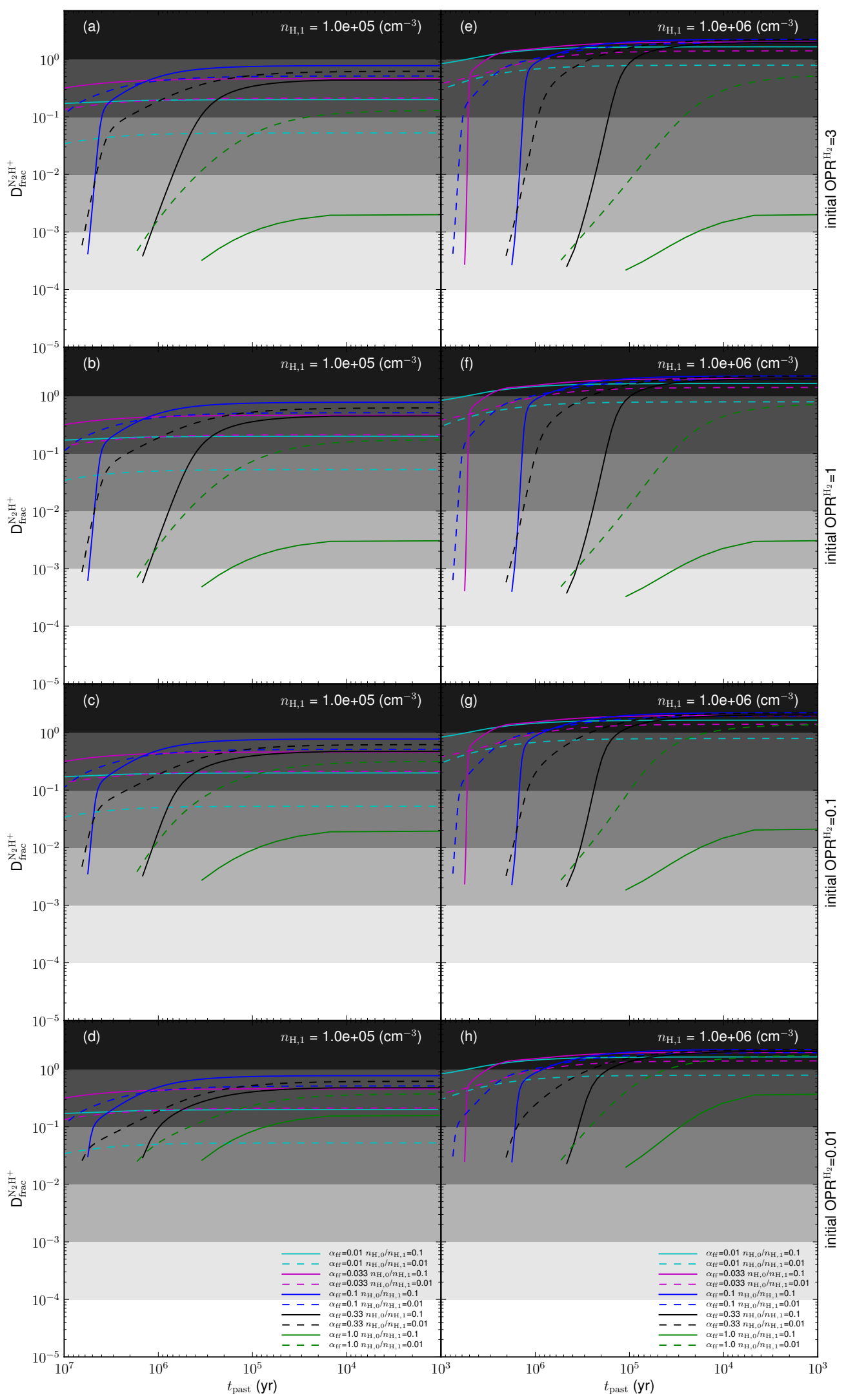

Figure 15. Same as Fig. 14 but starting with $f_{\mathrm{D}}=10$. 


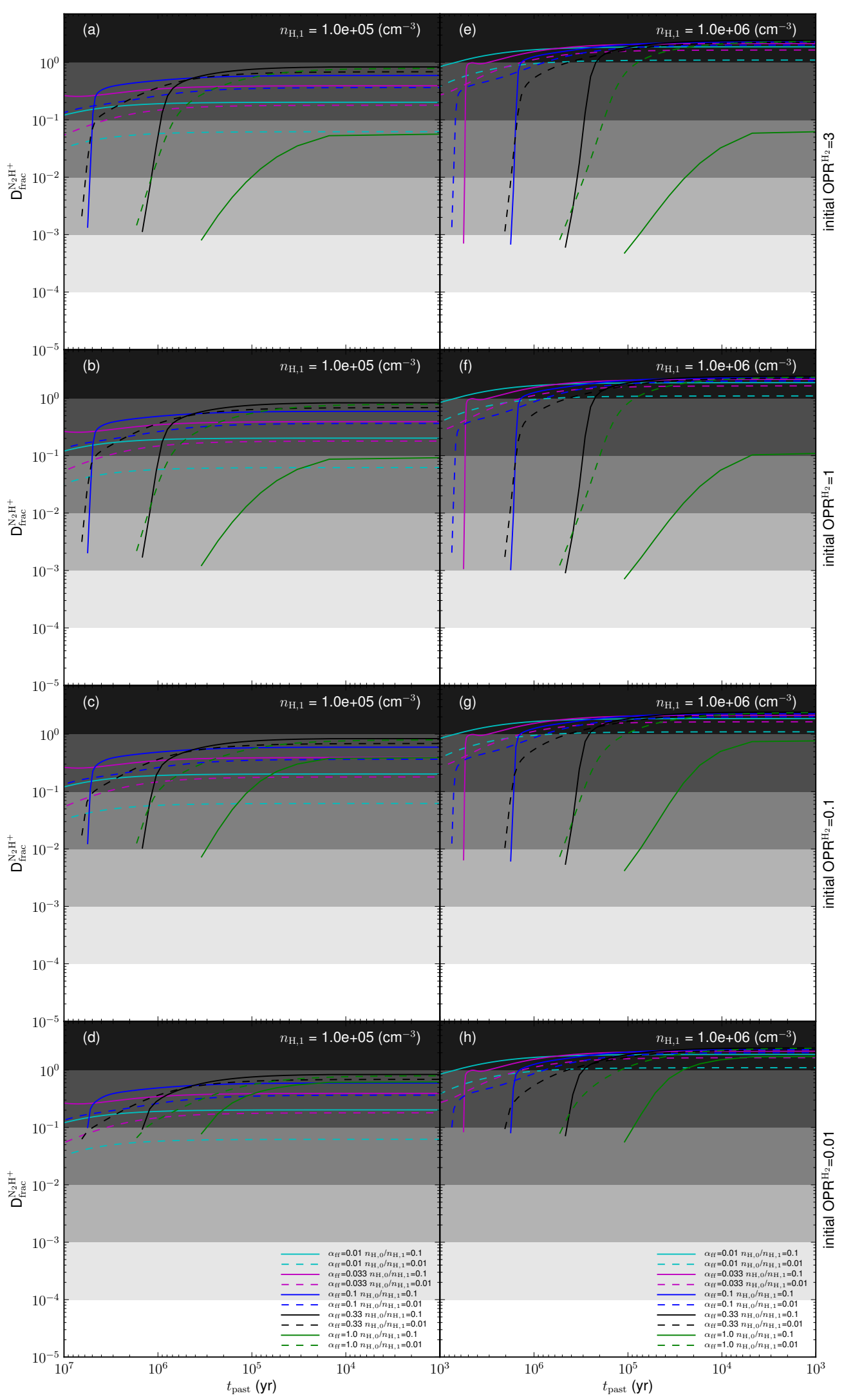

Figure 16. Same as Fig. 14 but starting with $f_{\mathrm{D}}=100$. 


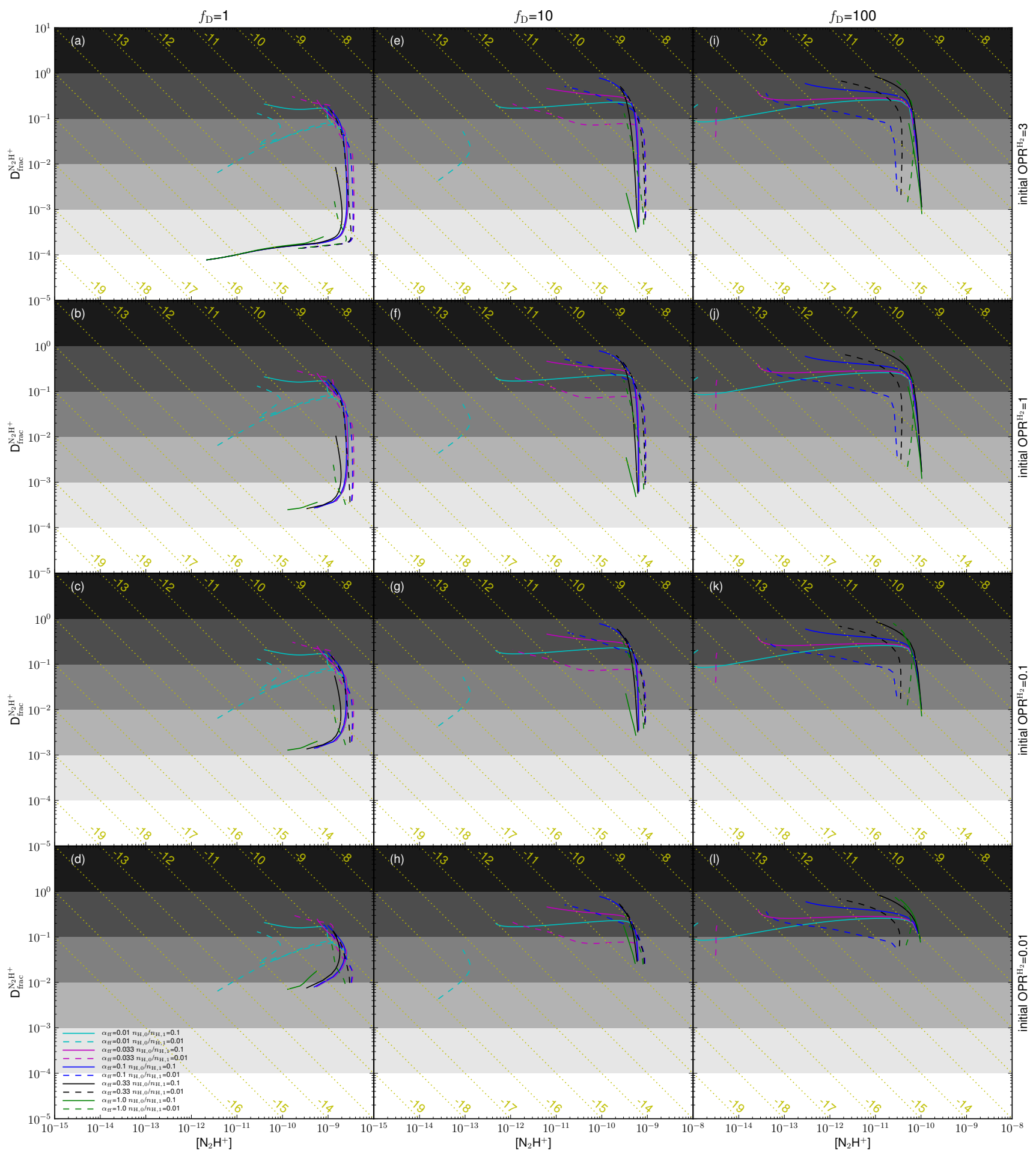

Figure 17. Relation between $D_{\text {frac }}^{\mathrm{N}_{2} \mathrm{H}^{+}}$and $\left[\mathrm{N}_{2} \mathrm{H}^{+}\right]$with different starting $f_{\mathrm{D}}$ (labeled on top) and OPR $\mathrm{H}_{2}$ (labeled on right). The modeled cores in this figure are from those in Figures $14\left[1516\right.$ having $n_{\mathrm{H}, 1}=10^{5} \mathrm{~cm}^{-3}$. The yellow dotted lines show constant $\left[\mathrm{N}_{2} \mathrm{D}^{+}\right]($the yellow numbers are indices with the base of 10). 


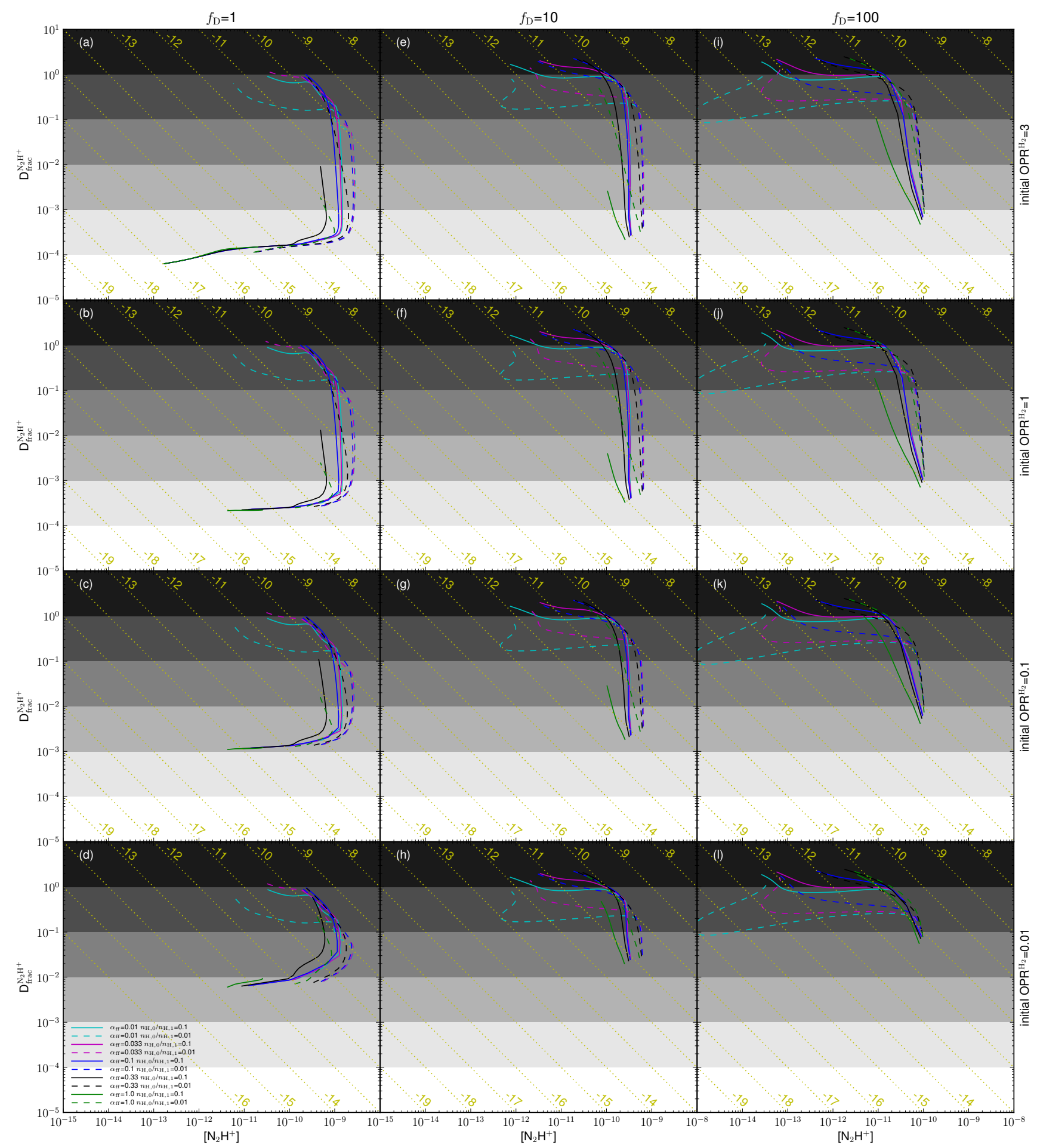

Figure 18. Same as Fig. 17 but with $n_{\mathrm{H}, 1}=10^{6} \mathrm{~cm}^{-3}$.

the $\mathrm{N}_{2} \mathrm{H}^{+}(1-0)$ line $), D_{\text {frac }}^{\mathrm{N}_{2} \mathrm{H}^{+}}=0.24($ Crapsi et al. 2005), the average number density is $n_{\mathrm{H}} \simeq 10^{6} \mathrm{~cm}^{-3}$ (Keto \& Caselli 2010), the temperature is about $6 \mathrm{~K}$ (Crapsi et

${ }^{4}$ We note that this observed $D_{\mathrm{fa}}^{\mathrm{N}_{2} \mathrm{H}^{+}}$value should be treated as a lower limit, as this is an average along the line of sight and it is well known that the $\mathrm{N}_{2} \mathrm{H}^{+}(1-0)$ emission is more extended than the $\mathrm{N}_{2} \mathrm{D}^{+}(2-1)$ emission (Caselli et al. 2002). Therefore, our estimates of the time scales are also lower limits. al. 2007), the cosmic ray ionization rate is $\simeq 1 \times 10^{-17} \mathrm{~s}^{-1}$ and the CO depletion factor is 100 (Keto \& Caselli 2010). With these parameters, we obtain the time to reach $D_{\text {frac }}^{\mathrm{N}_{2} \mathrm{H}^{+}}=0.2$ to be $4.2 \times 10^{5} \mathrm{yr}-2.6 \times 10^{5} \mathrm{yr}$, starting with $\mathrm{OPR}^{\mathrm{H}_{2}}=3-0.1$, respectively, i.e. between 9.6 and 5.9 times the current value of $t_{\mathrm{ff}}$. The corresponding value of $\mathrm{OPR}^{\mathrm{H}_{2}}$ is expected to be $\sim 3 \times 10^{-3}$

In cases where such a detailed analysis cannot be car- 
ried out, we can still derive some limits on the core deuteration timescale. For example, $D_{\text {frac }}^{\mathrm{N}_{2} \mathrm{H}^{+}} \gtrsim 0.1$ has been measured in low-mass pre-stellar cores (Crapsi et al. 2005. P09) and high-mass starless cores (Fontani et al. 2011, Miettinen et al. 2012), and there are currently no values of $D_{\text {frac }}^{\mathrm{N}_{2} \mathrm{H}^{+}}$observed to be greater than 1 . Starting from this, we examine all our simple models used in our parameter space exploration (3.4) to find how long it takes for $D_{\text {frac }}^{\mathrm{N}_{2} \mathrm{H}^{+}}$to reach 0.1 . The results are shown as the blue dashed lines in the 4th row of Fig. 6. The "missing" parts indicate conditions under which $D_{\text {frac }}^{\mathrm{N}_{2} \mathrm{H}^{+}}$fails to reach 0.1 . As one can see from the figure, to reach $D_{\mathrm{frac}}^{\mathrm{N}_{2} \mathrm{H}^{+}}>0.1$, the cores should be dense $\left(n_{\mathrm{H}} \gtrsim 5 \times 10^{4} \mathrm{~cm}^{-3}\right)$, cold $(T \lesssim 17 \mathrm{~K})$, at least moderately depleted $\left(f_{\mathrm{D}} \gtrsim 6\right)$, and with moderate $\mathrm{CR}$ ionization rates $\left(\zeta \lesssim 6 \times 10^{-17} \mathrm{~s}^{-1}\right)$. In all cases, the time to reach $D_{\text {frac }}^{\mathrm{N}_{2} \mathrm{H}^{+}}=0.1$ are longer than $t_{\mathrm{ff}}$. With moderate depletion $\left(f_{\mathrm{D}} \lesssim 100\right)$, the large $D_{\text {frac }}^{\mathrm{N}_{2} \mathrm{H}^{+}}(\gtrsim 0.1)$ is likely to indicate a large deuteration age $\left(\gtrsim\right.$ several $\left.t_{\mathrm{ff}}\right)$ for the observed starless cores. As shown in Figure 10 , these constraints can be somewhat relaxed if the starting $\mathrm{OPR}^{\mathrm{H}_{2}}$ values are small $(\lesssim 0.1)$ and the starting depletion factors large $\left(f_{\mathrm{D}} \gtrsim 100\right)$ (see also discussion in Pagani et al. 2011, and 4.3, below).

\subsection{Implications for magnetic support and comparison with the ambipolar diffusion time}

If contraction of starless cores is very slow compared to the local free-fall time, then this implies some form of pressure support is resisting collapse (see also Keto \& Caselli 2010). In cores that are more massive than the thermal Bonnor-Ebert mass, such as L1544 and the massive cores studied by Tan et al. (2013), this pressure support would need to be nonthermal, i.e. turbulence or magnetic fields. However, turbulence is expected to decay relatively quickly, within $\sim 1 t_{\text {ff }}$ (Stone et al. 1998 Mac Low et al. 1998), leaving magnetic fields as the favored option. This would imply core collapse occurs on the ambipolar diffusion timescale $t_{\mathrm{ad}}$ : the timescale for neutrals in dense cores with low ionization fractions to contract relative to the magnetic field (e.g. Tassis \& Mouschovias 2004).

The ambipolar diffusion timescale can be calculated using the expression $t_{\mathrm{ad}}=2.5 \times 10^{13} x(e)$ yr (Spitzer 1978; Shu et al. 1987), where $x(e)$ is the electron abundance relative to $n_{\mathrm{H}}$. Figure 6 plots $t_{\mathrm{ad}}$, to compare with $t_{\text {eq,90 }}\left(D_{\text {frac }}^{\mathrm{N}_{2} \mathrm{H}^{+}}\right)$and $t_{\mathrm{ff}}$. As $t_{\mathrm{ad}}$ is closely related to the ionization structure in core, high density and low $\zeta$ conditions reduce $t_{\mathrm{ad}}$, as shown in panels $(\mathrm{e}),(\mathrm{g}),(\mathrm{m})$ and (o). For fiducial conditions, the deuteration timescale is more similar to the local ambipolar diffusion timescale than to the free-fall time. Appreciating the caveats of estimates of deuteration timescales, discussed above, we conclude this is tentative, indirect evidence that magnetic fields are playing an important role in regulating starless core formation, and thus star formation.

\subsection{Comparison to Previous Studies}

There have been a number of discussions regarding the deuteration chemistry in pre-stellar cores (e.g. Pagani et al. 1992; Flower et al. 2006; P09; Sipilä et al. 2010; Parise et al. 2011, Wirström et al. 2012; Pagani et al. 2013). Compared with most of these previous studies, we have focussed on $D_{\text {frac,eq }}^{\mathrm{N}_{2} \mathrm{H}^{+}}$, using a more complete cold core chemistry network and with a larger and more systematic exploration of the parameter space of environmental conditions that help control the chemistry.

In the following, we compare our model with some of these works. Parise et al. (2011) benchmarked their results against that of P09 and Sipilä et al. (2010), but they do not show results for $D_{\text {frac }}^{\mathrm{N}_{2} \mathrm{H}^{+}}$. Both P09 and Sipilä et al. (2010) used Hugo et al. (2009) $\mathrm{H}_{3}^{+}+\mathrm{H}_{2}$ reaction system (including spin states and deuterium). Sipilä et al. (2010) also used dissociative recombination reactions from $\mathrm{P} 09$, but no elements heavier than $\mathrm{He}$ were considered. Taking this into consideration, we will only compare our models directly with P09 out of these three papers. We will also compare with Wirström et al. (2012). Note that although Aikawa et al. (2012) built a comprehensive chemical/dynamical model that included deuterium chemistry and followed the evolution of prestellar cores to the formation of protostars, since they do not include spin state chemistry, a direct comparison with our results cannot be made.

Our work can be compared most closely to that of P09, who included spin state chemistry and modeled the evolution of the abundance ratio of $\mathrm{N}_{2} \mathrm{D}^{+}$relative to $\mathrm{N}_{2} \mathrm{H}^{+}$ and discussed its use as a chemical clock. They used a modified version of the Nahoon code to model about 35 species and 400 reactions. They did not model $\mathrm{N}$ chemistry: in particular the abundance of $\mathrm{N}_{2}$ was a parameter in their modeling, so absolute abundances are not predicted. They developed a simple layered model for core structure that they compared to observations of the pre-stellar core L183. Based on the observed relatively low $D_{\text {frac }}^{\mathrm{N}_{2} \mathrm{H}^{+}}$in the center of the core, they concluded this central region must have only attained high density relatively recently. They estimated a minimum age of $\sim 2 \times 10^{5} \mathrm{yr}$, but suggested that it may not be that much older than this.

We ran our models with P09's choices of parameters, and compared with their Figs. 7, 8 \& 9. In particular, they used $T=7 \mathrm{~K}, \zeta=2 \times 10^{-17} \mathrm{~s}^{-1}$. Their dustto-gas mass ratio, grain radius, and dust grain density are the same as our fiducial model. They also used a fixed density and did not include time-dependent depletion/desorption. They set $\mathrm{CO}$ abundance to be $10^{-5}$ and $n_{\mathrm{H}}=1.4 \times 10^{5} \mathrm{~cm}^{-3}$ in the outer layer, and set $\mathrm{CO}$ abundance to be $10^{-6}$ and $n_{\mathrm{H}}=4.2 \times 10^{6} \mathrm{~cm}^{-3}$ in the inner layer. It is unclear whether they had leftover $\mathrm{C}$ and $\mathrm{O}$ atoms in their models. Here we simply assume all $\mathrm{C}$ and $\mathrm{O}$ were in $\mathrm{CO}$ in their models. Moreover, they were unclear about what initial $\left[\mathrm{N}_{2}\right]$ they used in the models (this was an input parameter of their models). We try our fiducial [N] in both runs (case 1). We also tried two more models with $\left[\mathrm{N}_{2}\right]=[\mathrm{CO}]$ value of P09 (case 2 ). We utilize $\mathrm{P} 09$ starting value of $\mathrm{OPR}^{\mathrm{H}_{2}}=3$.

The results of our models are summarized in Table 4 and compared with P09. We first look at the timescales. For the outer shell, the four timescales reported by our model are generally $\sim 2-3$ times that of P09 models. But for the inner shell our results are closer to those of P09. 
Recalling our parameter space exploration in Fig. 6, the depletion factor $f_{\mathrm{D}}$ has a strong effect on the equilibrium timescales. Since it is unclear what were the exact abundances used by P09 for C, N, O, the difference of the timescales in Table 4 could be due to differences in abundances, i.e. depletion factor.

The fact that our fiducial model is somewhat slower compared to P09 Figure 7 is likely due to their choice of stronger depletion. Recalling our Fig. 6, the high density in the P09 cores shortens the equilibrium timescale somewhat, but this is compensated by P09's choice of a slightly smaller $\zeta$. However, the large depletion factor can greatly shorten the equilibrium time. In P09 inner shell $[\mathrm{CO}]=10^{-6}$ (if they did not have leftover atomic $\mathrm{C}$ and $\mathrm{O}$ ), then this corresponds to $f_{\mathrm{D}}=146$ for $\mathrm{C}$ and $f_{\mathrm{D}}=360$ for $\mathrm{O}$ based on our choices for initial elemental abundances, which would greatly shorten the timescales if $f_{\mathrm{D}}$ for $\mathrm{N}$ is comparable to our models.

In P09 Figure 7, the equilibrium time for $D_{\mathrm{frac}}^{\mathrm{N}_{2} \mathrm{H}^{+}}$was $\sim 5$ times longer than the local instantaneous free-fall time. However, their conclusion was that the core did not reach the equilibrium. Our model predicts a much longer chemical equilibrium timescale compared to the local free-fall time (also dependent on $f_{\mathrm{D}}$ ). If we were to observe cores with the relevant high $D_{\text {frac }}^{\mathrm{N}_{2} \mathrm{H}^{+}}$, then their ages should be relatively old.

Therefore, in terms of $D_{\text {frac,eq }}^{\mathrm{N}_{2} \mathrm{H}^{+}}$and $t_{\text {eq,90 }}\left(D_{\text {frac }}^{\mathrm{N}_{2} \mathrm{H}^{+}}\right)$, our model agrees with P09 within a factor of 2-3. The major differences come from the assumption of initial depletion and core equilibrium state. These need to be constrained by observations.

Wirström et al. (2012) used a network with 4420 reactions, and their equilibrium time for $\mathrm{OPR}^{\mathrm{H}_{2}}$ is larger than $10^{6} \mathrm{yr}$, which is more similar to our value. They used $n_{\mathrm{H}}=2 \times 10^{6} \mathrm{~cm}^{-3}, T=10 \mathrm{~K}, A_{\mathrm{V}}>10 \mathrm{mag}$, $\zeta=3 \times 10^{-17} \mathrm{~s}^{-1}$, starting $\mathrm{OPR}^{\mathrm{H}_{2}}=3$. They allowed all neutral species (except for $\mathrm{H}_{2}, \mathrm{He}, \mathrm{N}$, and $\mathrm{N}_{2}$ ) to freezeout, but no desorption was considered. One thing to note is that we are not sure about what initial elemental abundances Wirström et al. (2012) used. They reference to Savage \& Sembach (1996) who reported elemental abundances in a variety of environments. We are not sure what specific initial abundances Wirström et al. (2012) used. So the comparison here is just qualitative.

\section{CONCLUSIONS}

We have presented a parameter space exploration of the deuterium fractionation process, in particular of $\mathrm{N}_{2} \mathrm{H}^{+}$, in conditions appropriate to starless dense cloud cores in different environments. An enhanced 3-atom reaction network is introduced. It was derived from a reduced chemical network extracted from the KIDA database to which Deuterium and spin state chemistry has been included. Reactions involving $\mathrm{H}_{3} \mathrm{O}^{+}$and its deuterated forms are introduced from Sipilä et al. (2013), to be able to reproduce results from the more comprehensive model of Sipilä et al. (2013). The effects of time-dependent depletion and dynamical density evolution have also been examined. Compared to previous studies (e.g., Pagani et al. 2009), our focus is on conditions that are also relevant for massive star formation, such as the massive starless cores observed by Tan et al. (2013). Our main results are as follows:
- Based on our fiducial modeling, the equilibrium value of $\left[\mathrm{N}_{2} \mathrm{D}^{+}\right] /\left[\mathrm{N}_{2} \mathrm{H}^{+}\right]$monotonically increases with increasing density (from $10^{3} \mathrm{~cm}^{-3}<n_{\mathrm{H}}<10^{7} \mathrm{~cm}^{-3}$ ), and decreasing $\mathrm{CR}$ ionization rate $\left(10^{-18} \mathrm{~s}^{-1}<\zeta<10^{-15}\right.$ $\left.\mathrm{s}^{-1}\right)$. With increasing temperature, the equilibrium $\left[\mathrm{N}_{2} \mathrm{D}^{+}\right] /\left[\mathrm{N}_{2} \mathrm{H}^{+}\right]$first moderately increases from $T \simeq 5 \mathrm{~K}$ to $T \simeq 13 \mathrm{~K}$, then decreases to $T \simeq 30 \mathrm{~K}$. With increasing freeze-out, the equilibrium $\left[\mathrm{N}_{2} \mathrm{D}^{+}\right] /\left[\mathrm{N}_{2} \mathrm{H}^{+}\right]$increases from $f_{\mathrm{D}} \simeq 1$ to $f_{\mathrm{D}} \simeq 1000$, but drops from $f_{\mathrm{D}} \simeq 2000$ to $f_{\mathrm{D}} \simeq 10^{6}$.

- When the gas temperature exceeds $\simeq 20 \mathrm{~K}$, the orthoto-para $\mathrm{H}_{2}$ ratio increases, reducing the deuterium fraction, so that warmer starless cores should display lower deuterium fractions (as found in high-mass star-forming regions by Fontani et al. 2011).

- The above findings are robust against changes in the initial elemental and molecular abundances.

- Constraints on core ages and collapse rates can be obtained if accurate measurements of $\left[\mathrm{N}_{2} \mathrm{D}^{+}\right] /\left[\mathrm{N}_{2} \mathrm{H}^{+}\right]$are made, coupled with observations of core density, temperature and $(\mathrm{CO})$ depletion structure. However, results can also depend on the cosmic ray ionization rate and the initial ortho-to-para ratio of $\mathrm{H}_{2}$.

- In the case of the well-known low-mass pre-stellar core L1544, we estimate that the gas within the central $3600 \mathrm{AU}$ has a deuteration age between $\simeq 6$ and 10 times the current local free-fall time, depending on the initial value of the ortho-to-para $\mathrm{H}_{2}$ ratio.

- More generally, to reproduce the typical deuterium fractions measured toward low-mass and massive prestellar cores $\left(\left[\mathrm{N}_{2} \mathrm{D}^{+}\right] /\left[\mathrm{N}_{2} \mathrm{H}^{+}\right] \gtrsim 0.1\right)$, the following physical parameters are needed: $n_{\mathrm{H}} \gtrsim 3 \times 10^{4} \mathrm{~cm}^{-3}, T \lesssim$ $17 \mathrm{~K}$, depletion factor $\gtrsim 6$, and cosmic ray ionization rate $\lesssim 10^{-16} \mathrm{~s}^{-1}$. In general, these values of deuterium fractions require timescales several times longer than the local free-fall timescale. With no initial depletion, the inclusion of time-dependent depletion/desorption has only a modest effect on these conclusions. Also with no initial depletion, models with dynamically evolving density, increasing by a factor of 10 , require collapse rates about 10 times slower than free-fall to reach the above levels of deuteration in cores with $n_{\mathrm{H}}=10^{6} \mathrm{~cm}^{-3}$. This suggests that dense cores with large deuterium fractions are dynamically old, which would likely require support against gravity to be provided by magnetic fields. For our fiducial model parameters, the timescale to reach deuteration equilibrium is similar to the expected ambipolar diffusion timescale, i.e., the collapse time of a magnetically subcritical core. The above conclusions can be avoided if the initial depletion factor is $\gtrsim 10$ (in which case rapidly collapsing cores could reach $\left.\left[\mathrm{N}_{2} \mathrm{D}^{+}\right] /\left[\mathrm{N}_{2} \mathrm{H}^{+}\right] \gtrsim 0.1\right)$, the cosmic ray ionization rate is very high $\left(\gtrsim 10^{-16} \mathrm{~s}^{-1}\right)$ or if the initial ortho-to-para ratio of $\mathrm{H}_{2}$ in the core is very small $(\lesssim 0.01)$, although this last condition itself would require the parental cloud to have a significant age.

\section{ACKNOWLEDGMENTS}

The authors acknowledge the continuous and fruitful interactions with Jorma Harju, and an anonymous referee for helping improve the manuscript. SK acknowledges support from Xueying Tang and an NRAO Student Observing Support grant. JCT acknowledges support from Univ. of Florida Research Opportunity Seed Fund 
Table 4

Comparison with P09 models of inner and outer shells.

\begin{tabular}{|c|c|c|c|c|c|c|}
\hline Model & $\begin{array}{l}\mathrm{OPR}_{\mathrm{eq}}^{\mathrm{H}_{2}} \\
\left(\times 10^{-4}\right)\end{array}$ & $\begin{array}{c}t_{\mathrm{eq}}\left(\mathrm{OPR}^{\mathrm{H}_{2}}\right) \\
\left(10^{6} \mathrm{yr}\right)\end{array}$ & $\begin{array}{c}t_{\mathrm{eq}, 90}\left(\mathrm{OPR}^{\mathrm{H}_{2}}\right) \\
\left(10^{6} \mathrm{yr}\right)\end{array}$ & $D_{\text {frac,eq }}^{\mathrm{N}_{2} \mathrm{H}^{+}}$ & $\begin{array}{c}t_{\mathrm{eq}}\left(D_{\mathrm{frac}}^{\mathrm{N}_{2} \mathrm{H}^{+}}\right) \\
\left(10^{6} \mathrm{yr}\right)\end{array}$ & $\begin{array}{c}t_{\mathrm{eq}, 90}\left(D_{\mathrm{frac}}^{\mathrm{N}_{2} \mathrm{H}^{+}}\right) \\
\left(10^{6} \mathrm{yr}\right)\end{array}$ \\
\hline outer shell (P09) ${ }^{a}$ & 0.99 & 0.80 & 0.69 & 0.71 & 0.84 & 0.54 \\
\hline outer shell $(\text { case } 1)^{\mathrm{b}}$ & 1.15 & 1.90 & 1.28 & 0.399 & 1.64 & 1.02 \\
\hline outer shell $(\text { case } 2)^{c}$ & 1.41 & 2.30 & 1.57 & 0.289 & 1.98 & 1.25 \\
\hline inner shell $(\mathrm{P} 09)^{\mathrm{a}}$ & 0.53 & 0.38 & 0.28 & 5.9 & 0.30 & 0.17 \\
\hline inner shell $(\text { case } 1)^{\mathrm{b}}$ & 0.326 & 0.575 & 0.340 & 2.24 & 0.465 & 0.234 \\
\hline inner shell $(\text { case } 2)^{c}$ & 0.299 & 0.515 & 0.298 & 2.96 & 0.415 & 0.200 \\
\hline
\end{tabular}

a The P09 values are read from their figures using the Dexter tool incorporated in A\&A online journal, for which we estimate $\sim 1 \%$ uncertainties.

$\mathrm{b}$ In this case we used $[\mathrm{N}]$ in our fiducial model.

c In this case we used $[\mathrm{N}]=[\mathrm{CO}]$ in P09.

and the Florida Space Inst. VW acknowldeges funding by the French INSU/CNRS program PCMI, the Observatoire Aquitain des Sciences de l'Univers and the European Research Council (ERC Grant 336474: 3DICE).

\section{REFERENCES}

Aikawa, Y., Wakelam, V., Hersant, F., Garrod, R. T., \& Herbst, E. 2012, ApJ, 760, 40

Bacmann, A., Lefloch, B., Ceccarelli, C., et al. 2003, ApJ, 585, L55

Bergin, E. A., Plume, R., Williams, J. P., \& Myers, P. C. 1999, ApJ, 512, 724

Bergin, E. A., \& Tafalla, M. 2007, ARA\&A, 45, 339

Bisschop, S. E., Fraser, H. J., Öberg, K. I., van Dishoeck, E. F., \& Schlemmer, S. 2006, A\&A, 449, 1297

Bodenheimer P.H., 2011, Principles of Star Formation. Springer, Berlin

Brünken, S., Sipilä, O., Chambers, E. T., et al. 2014, Nature, 516, 219

Butler, M. J., \& Tan, J. C. 2012, ApJ, 754, 5

Caselli, P. 2002, Planet. Space Sci., 50, 1133

Caselli, P., Walmsley, C. M., Terzieva, R., \& Herbst, E. 1998, ApJ, 499, 234

Caselli, P., Walmsley, C. M., Tafalla, M., Dore, L., \& Myers, P. C. 1999, ApJ, 523, L165

Caselli, P., Walmsley, C. M., Zucconi, A., et al. 2002, ApJ, 565, 344

Caselli, P., Vastel, C., Ceccarelli, C., et al. 2008, A\&A, 492, 703

Ceccarelli, C., Hily-Blant, P., Montmerle, T., et al. 2011, ApJ, 740, L4

Crabtree, K. N., Indriolo, N., Kreckel, H., Tom, B. A., \& McCall, B. J. 2011, ApJ, 729, 15

Crapsi, A., Caselli, P., Walmsley, C. M., et al. 2005, ApJ, 619, 379

Crapsi, A., Caselli, P., Walmsley, M. C., \& Tafalla, M. 2007, A\&A, 470, 221

Dalgarno, A. 2006, Proceedings of the National Academy of Science, 103, 12269

Dalgarno, A., \& Lepp, S. 1984, ApJ, 287, L47

Draine, B. T., \& Sutin, B. 1987, ApJ, 320, 803

Draine, B. T. 2011, Physics of the Interstellar and Intergalactic Medium by Bruce T. Draine. Princeton University Press, 2011. ISBN: 978-0-691-12214-4

Emprechtinger, M., Caselli, P., Volgenau, N. H., Stutzki, J., \& Wiedner, M. C. 2009, A\&A, 493, 89

Flower, D. R., Pineau Des Forêts, G., \& Walmsley, C. M. 2006, A\&A, 449, 621

Fontani, F., Caselli, P., Crapsi, A., et al. 2006, A\&A, 460, 709

Fontani, F., Zhang, Q., Caselli, P., \& Bourke, T. L. 2009, A\&A, 499, 233

Fontani, F., Palau, A., Caselli, P., et al. 2011, A\&A, 529, L7

Friesen, R. K., Di Francesco, J., Myers, P. C., et al. 2010, ApJ, 718,666

Garrod, R. T., Wakelam, V., \& Herbst, E. 2007, A\&A, 467, 1103

Guelin, M., Langer, W. D., Snell, R. L., \& Wootten, H. A. 1977, ApJ, 217, L165
Hasegawa, T. I., \& Herbst, E. 1993, MNRAS, 261, 83

Hasegawa, T. I., Herbst, E., \& Leung, C. M. 1992, ApJS, 82, 167

Hernandez, A. K., Tan, J. C., Caselli, P., et al. 2011, ApJ, 738, 11

Hily-Blant, P., Walmsley, M., Pineau Des Forêts, G., \& Flower, D. 2010, A\&A, 513, A41

Hugo E., Asvany O., Schlemmer S., 2009, J. Chem. Phys., 130, 164302

Keto, E., \& Caselli, P. 2010, MNRAS, 402, 1625

Le Petit, F., Roueff, E., \& Le Bourlot, J. 2002, A\&A, 390, 369

Lesaffre, P., Belloche, A., Chièze, J.-P., \& André, P. 2005, A\&A, 443,961

Li, X., Heays, A. N., Visser, R., et al. 2013, A\&A, 555, A14

Mac Low, M.-M., Klessen, R. S., Burkert, A., \& Smith, M. D. 1998, Physical Review Letters, 80, 2754

Maret, S., \& Bergin, E. A. 2007, ApJ, 664, 956

Miettinen, O., Harju, J., Haikala, L. K., \& Juvela, M. 2012, A\&A, 538, A 137

Oka, T. 2004, Journal of Molecular Spectroscopy, 228, 635

Oliveira, C. M., Hébrard, G., Howk, J. C., et al. 2003, ApJ, 587, 235

Pagani, L., Salez, M., \& Wannier, P. G. 1992, A\&A, 258, 479

Pagani, L., Vastel, C., Hugo, E., et al. 2009, A\&A, 494, 623

Pagani, L., Roueff, E., \& Lesaffre, P. 2011, ApJ, 739, L35

Pagani, L., Lesaffre, P., Jorfi, M., et al. 2013, A\&A, 551, A38

Parise, B., Belloche, A., Du, F., Güsten, R., \& Menten, K. M. 2011, A\&A, 526, A31

Pillai, T., Wyrowski, F., Carey, S. J., \& Menten, K. M. 2006, A\&A, 450,569

Pillai, T., Wyrowski, F., Hatchell, J., Gibb, A. G., \& Thompson, M. A. 2007, A\&A, 467, 207

Pillai, T., Caselli, P., Kauffmann, J., et al. 2012, ApJ, 751, 135

Pineau des Forets, G., Flower, D. R., \& McCarroll, R. 1991, MNRAS, 248, 173

Ragan, S. E., Bergin, E. A., \& Wilner, D. 2011, ApJ, 736, 163

Shu, F. H., Adams, F. C., \& Lizano, S. 1987, ARA\&A, 25, 23

Sipilä, O., Hugo, E., Harju, J., et al. 2010, A\&A, 509, A98

Sipilä, O., Caselli, P., \& Harju, J. 2013, A\&A, 554, A92

Spitzer L., Jr, 1978, Physical Processes in the Interstellar Medium. Princeton Univ. Press, Princeton, NJ

Stone, J. M., Ostriker, E. C., \& Gammie, C. F. 1998, ApJ, 508, L99

Tan, J. C., Kong, S., Butler, M. J., Caselli, P., \& Fontani, F. 2013, ApJ, 779, 96

Tan, J. C., Beltran, M. T., Caselli, P., et al. 2014, arXiv:1402.0919

Tassis, K., \& Mouschovias, T. C. 2004, ApJ, 616, 283

Troscompt, N., Faure, A., Maret, S., et al. 2009, A\&A, 506, 1243

van der Tak, F. F. S., \& van Dishoeck, E. F. 2000, A\&A, 358, L79

Vastel, C., Caselli, P., Ceccarelli, C., et al. 2012, A\&A, 547, A33

Wakelam, V., \& Herbst, E. 2008, ApJ, 680, 371

Wakelam, V., Herbst, E., Loison, J.-C., et al. 2012, ApJS, 199, 21

Walmsley, C. M., Flower, D. R., \& Pineau des Forêts, G. 2004,

A\&A, 418, 1035

Ward-Thompson, D., Motte, F., \& Andre, P. 1999, MNRAS, 305, 143

Williams, J. P., \& McKee, C. F. 1997, ApJ, 476, 166

Wirström, E. S., Charnley, S. B., Cordiner, M. A., \& Milam,

S. N. 2012, ApJ, 757, L11

Wootten, A., Snell, R., \& Glassgold, A. E. 1979, ApJ, 234, 876 